\title{
A review of Electrohydraulic Independent Metering Technology
}

\begin{abstract}
The subject of this paper is the review of advanced technology used in hydraulic systems. The technology in question is termed Independent Metering (IM); this is used in hydraulically driven mobile machinery, such as agricultural, construction, municipal, and forestry vehicles. The idea behind the concept is to modify the connection between the actuator, which could be a cylinder or a motor, and a flow control valve. Traditionally, spool hydraulic valves were used to control the fluid flow into and out of hydraulic actuators. This keeps the meter-in and the meter-out of the actuator mechanically connected due to the construction of these valves. This connection makes the control system blind to pressure changes in one of the hydraulic chambers in the actuator. This, in turn, reduces the overall system controllability. It also increases energy losses, especially under an overrunning load. These two main weaknesses led researchers to break this mechanical connection and get into a new technology with different characteristics. The proposed technology was called Independent Metering. New and more complex control techniques can now be applied to the hydraulic systems using this technology that were not possible before or could be applied to more conventional servo design. This paper reviews Independent Metering (IM) and the technologies used or developed in this field to date. The paper reviews the state of art hydraulic technologies and indicates the links between them and IM. It also reviews the different types of hydraulic valves used when implementing IM. This review also discusses some control algorithms, IM layouts, IM challenges, and identifies where further improvements may be achieved.
\end{abstract}

Preprint submitted to Journal of $E^{A} T_{E} X$ Templates

July 16, 2019 


\section{Introduction}

Hydraulic systems are important elements that indirectly contribute to the quality of human life. They are heavily used for a variety of applications ranging from constructional to industrial, military, aerospace, and earth moving 5 applications due to their unique and valuable characteristics. Compared to electrical actuators, hydraulic drives are characterized by high load capabilities, high power to weight ratio and robustness [1] and [2]. They are effective in applications with abrupt loading, frequent stops and variations. However, hydraulic drives still suffer from some shortcomings, such as energy losses and nonlinearities which makes the control system more challengeable [2]. These nonlinearities are largely due to friction and hysteresis, amongst other causes [3. Figure 1 illustrates the losses for a mobile load-sensing hydraulic system. About $30 \%$ of the energy losses are due to the traditional valves.

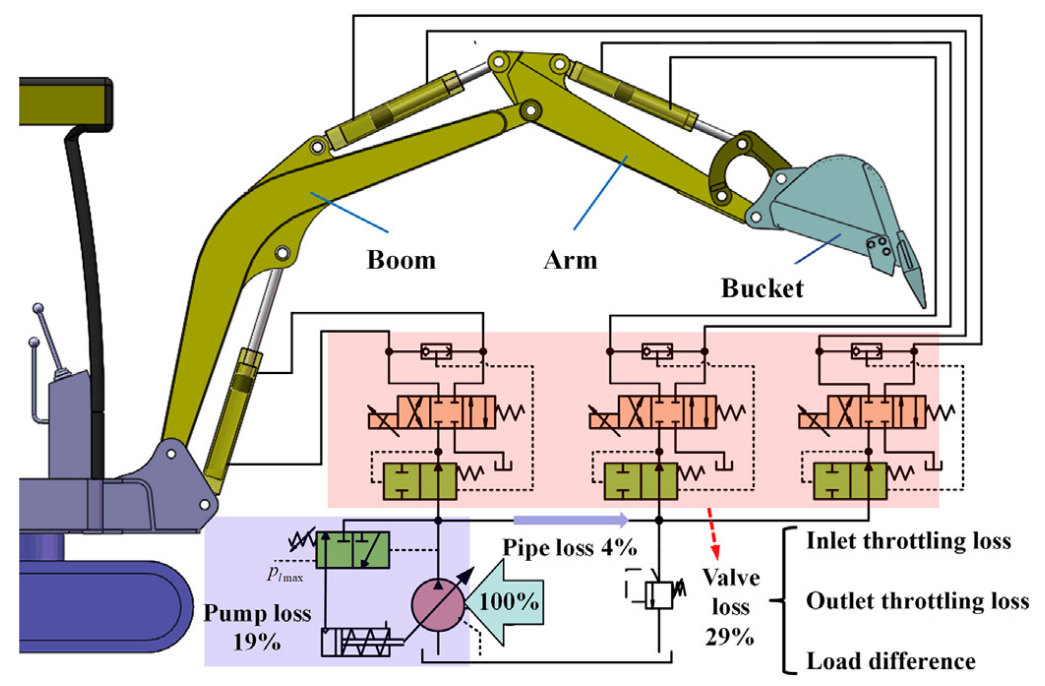

Figure 1 Losses in a hydraulic mobile machine controlled by traditional spool valve and variable displacement valve [4].

Hydraulic individualization methodology is used to improve power density, robustness and flexibility. Individualization can be split into displacement and valve control [5]. Typical displacement individualization is shown in Figure 2. 


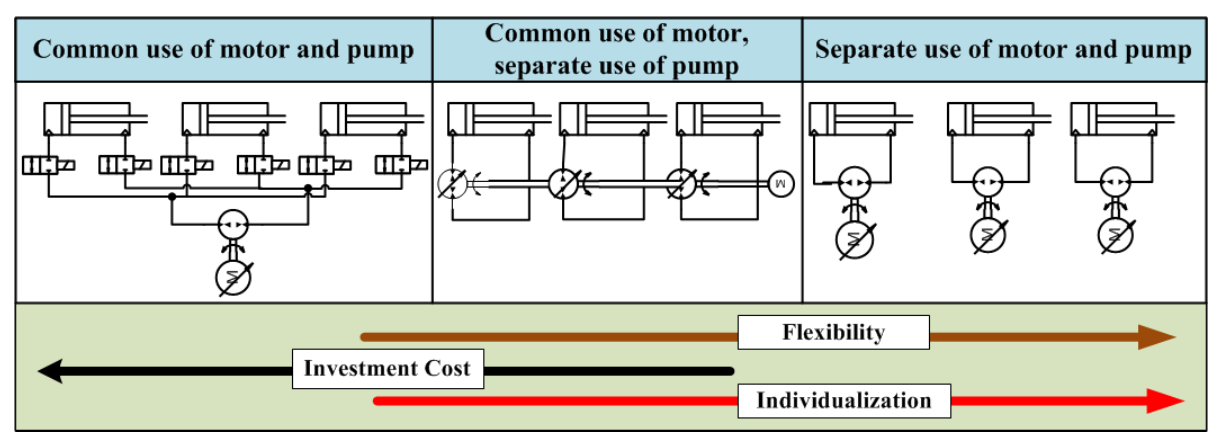

Figure 2 Illustrates different types of hydraulic drives individualization based on the use of pump and motor [5].

The most common technique, also the lowest level, was one pump feeding several actuators. This approach is common in injection and moulding machines [6. The second technique is mainly used in hydraulically driven mobile machines. A Green Wheel Loader was investigated by the Institute of Fluid Power at Dresden University of technology [7, and a (DC S-P) hybrid mini excavator was improved at Maha Fluid power research centre at Purdue University [8], both implementing this second method. The third or the last method, shown in Figure 2, is used in high power applications where every actuator has its own 25 pump. These systems main characteristics are lower energy consumption rates, hence better fuel economy and fewer greenhouse gases. Their main drawbacks are slower dynamics compared to servo actuators [2]. Iterations were established to improve the response of this method using electronics similar to what in [9] and [10].

Regarding individualization using hydraulic control valves, three main types of individualization are summarized in Figure 3 . 


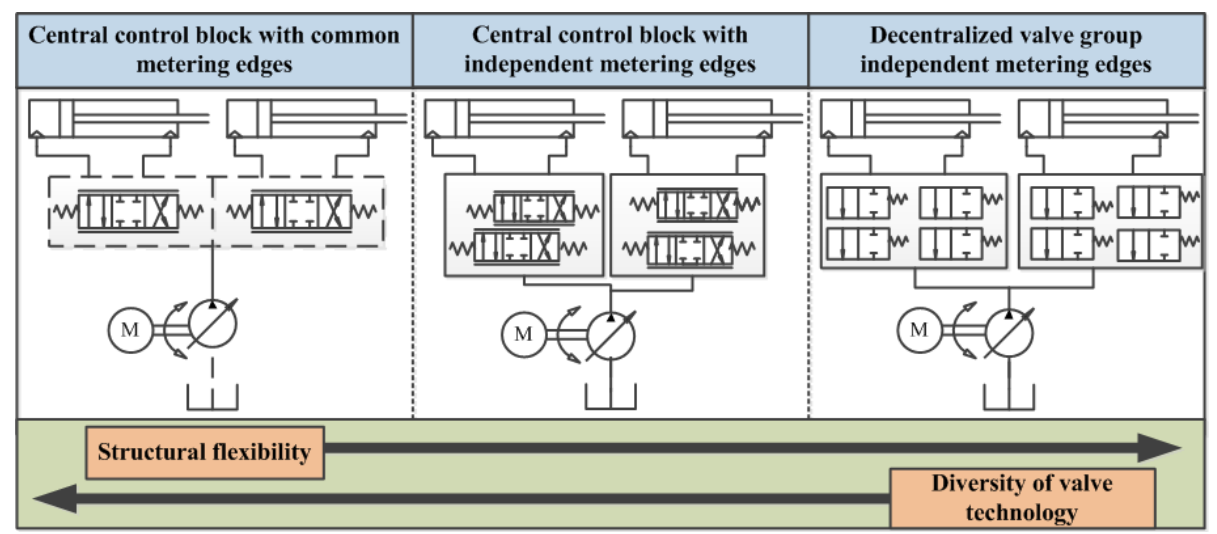

Figure 3 Hydraulic drive individualization based on valve structure, it shows the flexibility could be obtained using independent metering and comparing to common metering edge [5].

The first approach is a common metering edge, which is the traditional directional flow control approach. Each actuator is controlled by one valve. Due to the mechanical connection between the metering edges of the actuator using traditional spool valves, the system has one degree of freedom which means that the pressure of one cylinder chamber is controlled [11. This configuration limits the system flexibility, but it increases the robustness [12. To improve the efficiency and the energy saving, the trend was to break the mechanical connection between the meter-in and the meter-out edges, which is why it is called independent metering. Different terms are used for IM as separate metering, programmable valves, multifunctional valves and separate meter-in separate meter-out control [13, 14, 15]. It is termed by programmable because it changes the control system from the hydro-mechanical concept into an intelligent control system that relies on software. Breaking the mechanical connection 45 leads to many advantages and disadvantages as concluded from [16, 17, 18].

The main advantages are:

1. Independent control of inlet and outlet orifices.

2. Increased energy efficiency by allowing individual control paths or modes. This was proved for the excavator manipulator in [19]. 
4. Avoiding cavitation during a pulling load.

5. Flexible system configuration.

6. Transfer of functionality from hardware to software.

7. An ability to apply advanced control methods.

The main disadvantages are as follows:

1. Increased components costs.

2. Complex controllers are required.

3. Switching between modes cause sudden change in the velocity.

4. Difficulty of pressure compensator integration [20].

60

Research on IM systems has been conducted at different institutes and companies with various approaches. Figure (4) is a general statistical chart indicating the up to date developments in the IM. The main scientific institutes developing the intelligent hydraulic systems and precisely IM technology are summarized in Table (1). The numbers of researches and patents developed by these institutes and companies are illustrated in Figure (5). The first improvement was quantities decoupling [21. Many techniques were improved for decoupling such as LQ technique and pressure feedback [8] and [22]. The effects of feedback linearization and open loop control were investigated in [23] and 24. Besides, adaptive control, which is an important field, was used for these systems [13, 25] and [26]. On the other hand, Tabor developed a quasi-static mathematical technique for IM 27. Improving this model was by inserting continuous mode switching 28. The next significant improvement of the IM technology was done by introducing the digital hydraulics [29]. Every consumer, actuator, is actuated by four digital fluid control unit (DFCU) which contains 75 an array of on/off valves [30. There was also a new system based on a hybrid 
concept such as STEAM [8] and [31]. STEAM system relies on three pressure lines, high, medium and tank. Changing between these pressure lines pursues the functional decoupling of IM which will be explained in section 3 32.

This paper reviews different aspects related to IM technology. It includes the review of the different state of the art in these systems where the independent metering technology is discussed in more details. Besides, different IM valves configurations, operation modes, and switching techniques are reviewed. It also clarifies the main challenges facing the IM technology and the possible solutions to overcome them. Finally, IM control systems, layouts, and algorithms are reviewed in this paper.

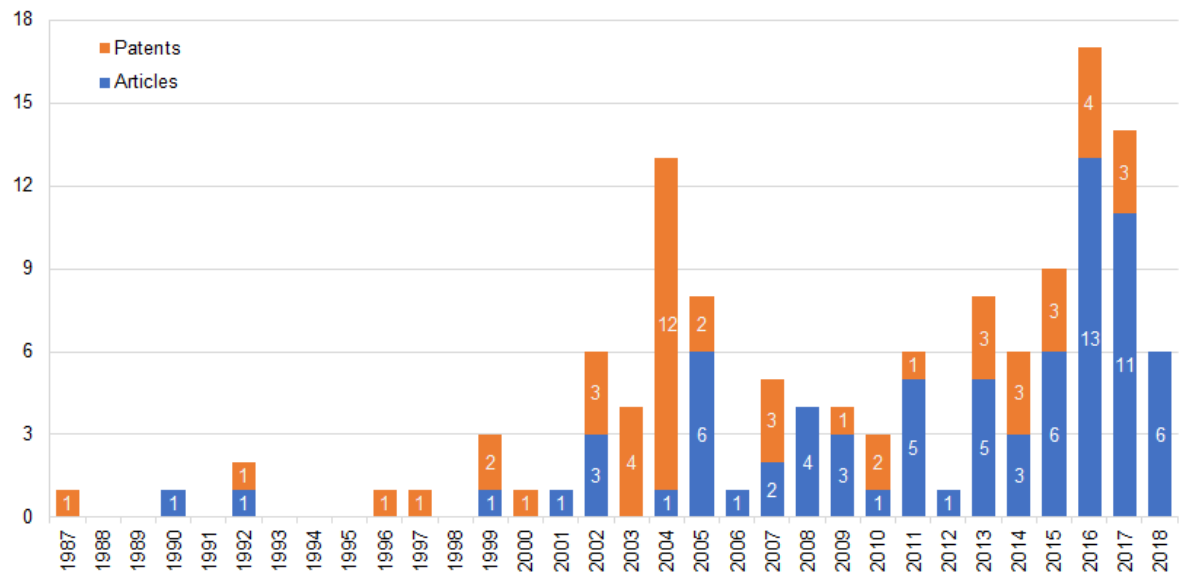

Figure 4 Number of studies related to IM in the last three decades. 
Table 1 Main academic institutes developing IM.

\begin{tabular}{|c|c|c|}
\hline University & Research Trends & Sample of Studies \\
\hline $\begin{array}{l}\text { Institute of Hydraulic and } \\
\text { Automation at Tampere } \\
\text { University of Technology, } \\
\text { Finland. }\end{array}$ & $\begin{array}{l}\text { 1. Digital Hydraulic. } \\
\text { 2. Hydraulic Manipulation } \\
\text { Development. }\end{array}$ & $\begin{array}{l}\text { 1. IM Hydraulic System } \\
{[33,34,35 .} \\
\text { 2. Hydraulic Manipula- } \\
\text { tions 36]. }\end{array}$ \\
\hline $\begin{array}{lr}\text { Institute of } & \text { Fluid and } \\
\text { Mechatronics } & \text { Systems } \\
\text { at Linkoping } & \text { University, } \\
\text { Sweden. } & \end{array}$ & $\begin{array}{l}\text { 1. IM and Load Sensing } \\
\text { System. } \\
\text { 2. Hybrid Hydraulic. } \\
\text { 3. Real-Time-Simulation } \\
\text { of Hydraulic Systems. }\end{array}$ & $\begin{array}{l}\text { 1. Load Sensing with IM } \\
\text { [37, 38, 39]. }\end{array}$ \\
\hline $\begin{array}{l}\text { Institute of Fluid power } \\
\text { at Dresden University of } \\
\text { Technology, Germany. }\end{array}$ & 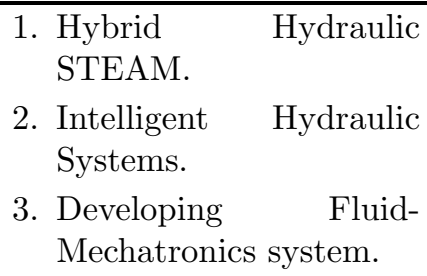 & $\begin{array}{l}\text { 1. Independent Metering } \\
\text { and Decentralization for } \\
\text { Energy Saving [40, 5]. } \\
\text { 2. Hybrid Hydraulic Sys- } \\
\text { tem [32]. }\end{array}$ \\
\hline $\begin{array}{l}\text { The George W. Woodruff } \\
\text { School of Mechanical En- } \\
\text { gineering at Georgia Insti- } \\
\text { tute of Technology, USA. }\end{array}$ & $\begin{array}{l}\text { 1. Hydraulics Component } \\
\text { Development. } \\
\text { 2. Intelligent Control of } \\
\text { Hydraulic Manipulators }\end{array}$ & $\begin{array}{l}\text { 1. Poppet Valve Develop- } \\
\text { ments [41, 42, 43]. } \\
\text { 2. Independent Metering } \\
\text { System [44, 45]. }\end{array}$ \\
\hline $\begin{array}{l}\text { State Key Laboratory of } \\
\text { Fluid Power and Mecha- } \\
\text { tronics System, Zhejiang } \\
\text { University, China. }\end{array}$ & $\begin{array}{l}\text { 1. Development of In- } \\
\text { dependent Metering } \\
\text { Switching Methods. } \\
\text { 2. Hydraulic Drive Motion } \\
\text { Improvement. }\end{array}$ & $\begin{array}{l}\text { 1. Independent Metering } \\
\text { Mode Switching [46]. } \\
\text { 2. Energy saving 47, 48. }\end{array}$ \\
\hline $\begin{array}{l}\text { Maha Fluid Power Center, } \\
\text { Purude University, USA. }\end{array}$ & $\begin{array}{l}\text { 1. Hydraulic Components } \\
\text { Modelling. } \\
\text { 2. Mobile Hydraulic Sys- } \\
\text { tems } \\
\text { 3. Noise Control. }\end{array}$ & $\begin{array}{l}\text { 1. Load Sensing with IM } \\
\text { [49, 50]. } \\
\text { 2. Modelling of Compo- } \\
\text { nents [51]. }\end{array}$ \\
\hline $\begin{array}{l}\text { Key Lab of Advanced } \\
\text { Transducers and Intelli- } \\
\text { gent System , Taiyuan } \\
\text { University, China. }\end{array}$ & $\begin{array}{l}\text { 1. Hydraulic Manipulation } \\
\text { System. } \\
\text { 2. Modelling of Hydraulic } \\
\text { System }\end{array}$ & $\begin{array}{l}\text { 1. Independent } \\
\text { Performance } \\
52\end{array}$ \\
\hline $\begin{array}{l}\text { School of Mechanical and } \\
\text { Automotive Engineering, } \\
\text { University of Ulsan, South } \\
\text { Korea. }\end{array}$ & $\begin{array}{l}\text { 1. Independent Metering } \\
\text { Implementation. } \\
\text { 2. Hydraulic Manipulation } \\
\text { Energy } \\
\end{array}$ & $\begin{array}{l}\text { 1. IM Systems [53]. } \\
\text { 2. IM Energy Saving [54]. }\end{array}$ \\
\hline $\begin{array}{l}\text { Institute of } \\
\text { Engineering, } \\
\text { USA. }\end{array}$ & 1. Dynamic and Control & 1. Valve Performance [55]. \\
\hline
\end{tabular}




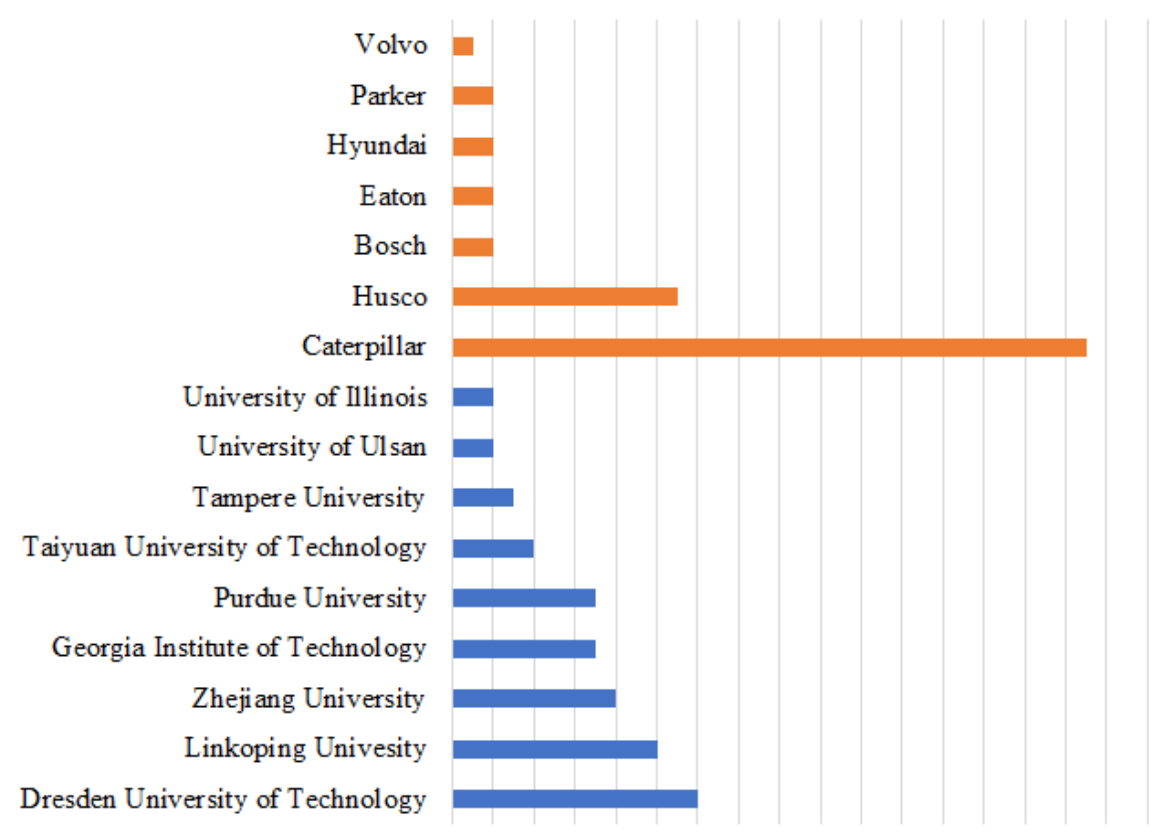

Figure 5 Contribution of the leading research institutes and companies to the IM development.

\section{State of the art}

As indicated in [56], the three main requirements to improve hydraulic machines efficiency are as follows:

1. Reducing throttle losses,

2. Avoiding an inefficient operating point,

3. Recovering potential energy.

Different methods were used to satisfy these requirements. They are load sensing (LS), digital drive, holistic systems and independent metering. LS is one of the most famous systems in hydraulic applications [57. It was mainly designed to save energy by producing the required amount of flow rate or pressure for the consumer [58. This pressure is produced by a variable displacement pump 
(VDP) based on the highest actuator pressure feedback [59]. The conventional LS systems were hydro-mechanical. The shortcomings of this traditional system are the poor damping and inconvenient performance 60, 61. The insertion of electronics was performed by Casappa and Wlviol about 30 years ago 62. An example of practical implementation of electronic load sensing control by HUSCO International, Inc [63. LS systems can be split into two main categories, Open-Centre (OC) and Closed Centre (CC) Hydraulic systems. The OC uses a fixed displacement pump, and the CC uses a variable displacement pump 64. The former has more losses than the latter especially when the load pressure is high and the required flow rate is small 65. Adding a pressure compensator to the CC leads to the load sensing pressure compensated LSPC technique which is shown in Figure 6. The pressure compensator reduces the influence of the pressure to the controlled flow. The LSPC's drawbacks are oscillations and a pressure margin. Oscillations are produced in the mechanical system by the compensator due to the produced poor damping and increased dynamic complexity. The pressure margin is an extra pressure needed to be produced by the pump. The pump pressure should be more than the demand from the most loaded actuator. This margin keeps the pressure level higher than the pressure drop by the pressure compensator [12.

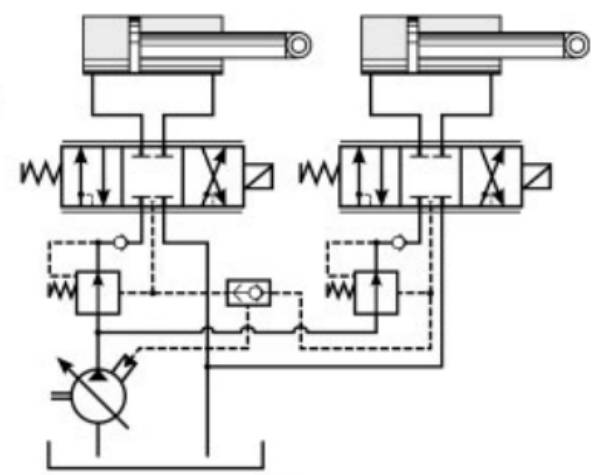

Figure 6 Load sensing pressure compensated circuit where a variable displacement pump uses the pressure feedback to produce the required flow and pressure 12.

Conventional spool valves controlled load sensing system still suffers from 
throttle losses due to the mechanical connection between the inlet and the outlet [29]. This connection is illustrated in Figure 7

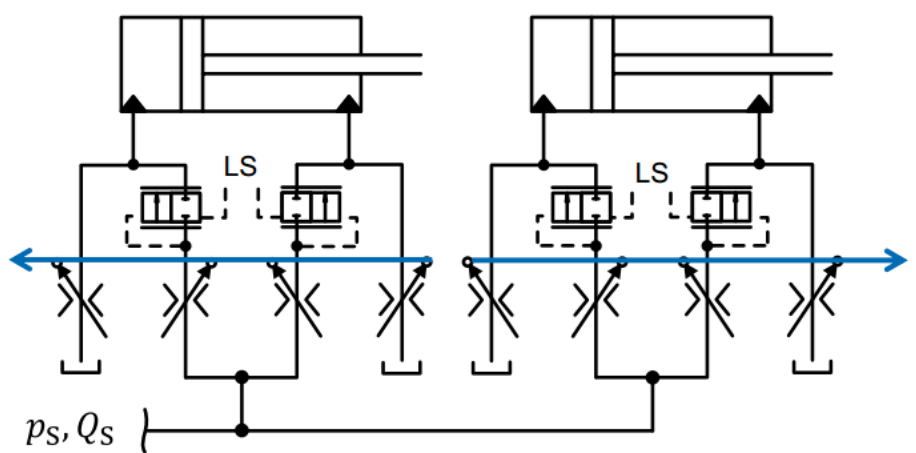

Figure 7 The mechanical connections between the actuator meter-in and meter-out due to the traditional spool valves. LS represents the load sensing signal, Ps and Qs represent the main pressure and flow sources from the pump [12].

To reduce these losses the mechanical connection should be broken and this leads to the IM technology [66]. Combining both systems IM and LS is a suitable approach to save more energy and produce better controllability. It also transfers functionality from hardware to software, fasts the work cycle time, and implements electronically tunable operation modes [67, 4.

An advanced practical implementation using both systems was developed by Caterpiller Inc [68. Also, an algorithm was developed for the combined systems when check valves are not used between the valve arrangement and the pump [69, and an algorithm when the required velocity flow is more than the pump flow [70].

Digital hydraulic is a term describing the digitalization of hydraulics. The main idea is to replace the continuous variable with a discrete one. This covers different hydraulic equipment such as valves, motors, accumulators and pumps [71. For example, a digital pump-motor was used to implement a digital hydraulic power management system (DHPMS) [72]. A Digital Flow Control Unit (DFCU) is a unit that connects a group of on/off valves in a parallel layout and their response is presented proportionally. These valves are not prone to 
leakage, are reliable and are insensitive to oil contamination. Using the digital valves to IM system adds extra advantages to the traditional IM that uses poppet or spool valves [73. Digital hydraulics can be controlled by three main techniques as follows [29] and [74]:

1. Pulse number modulation (PNM).

2. Pulse code modulation (PCM).

3. Fibonacci number.

The main drawbacks of digital fluid systems are as follows:

1. Larger overall size comparing with traditional valves.

2. The cost which depends on the application.

3. Noise and pressure peaks.

The Institute of fluid power drive and controls in Aachen produced a new configuration for hydraulic excavator called STEAM. It aims to reduce the valve control losses as well as the engine losses by hybrid architecture [32] and [75]. follows:

1. Using a constant pressure system saves more energy.

2. Using an intermediate pressure line reduces throttle losses.

3. Availability of regeneration and recuperation increases the energy efficiency.

The architecture of STEAM system offers the following advantages as follows:

1. Constant pressure system enables a fixed-point operation for the internal combustion engine (ICE) in the machine.

2. Using three pressure lines increases the number of operating state for each cylinder. 
The main drawback of STEAM systems is poor controllability as the system is based on different pressure lines [76], which produces high oscillation during switching between the pressure levels [57].

The mentioned technologies aim to save more energy and they intersect

with IM. For example, IM was implemented using the digital hydraulic concept. STEAM systems can be applied by using IM. Also, a variable displacement pump can be used with IM. To finish, independent metering, as mentioned before, is based on breaking the mechanical connections between metering ports. This requires different valves rather than the traditional spool type.

\section{IM valves}

Generally, hydraulic valves used to implement IM can be classified into 3/3 and $2 / 2$ valves [8]. These are used to make different forms of decoupling between the input and the output. Decoupling can be mechanical or functional, as summarized in Figure 8 [5]. The mechanical decoupling is based on changing the 175 valve kind from $3 / 3$ or $4 / 3$ into $2 / 2$ proportional valves which lead to different configurations of IM. The functional decoupling relies on the switching and proportional valves, where the functionality depends on the switching valve direction combined with flow controlled by proportional valves.

The first iteration to implement IM using $4 / 3$ valves was by Monsun-Tison [77, and the system called MONTI. An example of IM configuration using two $4 / 3$ valves is Caterpiller patent [78. Then, application of $3 / 3$ valves was introduced by EATON company. 2/2 valves were developed by many companies such as Deere, Moog and Caterpillar [79]. These valves are cartridge poppets and are widely used for IM control. The schematic design of the Valvistor valve is shown in Figure 9. Its work principle is similar to the electronic transistor where the pilot circuit drive a larger main flow. The main difference between $P_{b}$ and $P_{a}$ generated by $Q_{p}$ moves $m_{m}$ which is the main poppet for a distance $x_{m}$. The pilot stage which contains Pulse Width Modulation (PWM) solenoid controls the $Q_{p}$ flow. A block arrangement contains four valves for every actuator was 


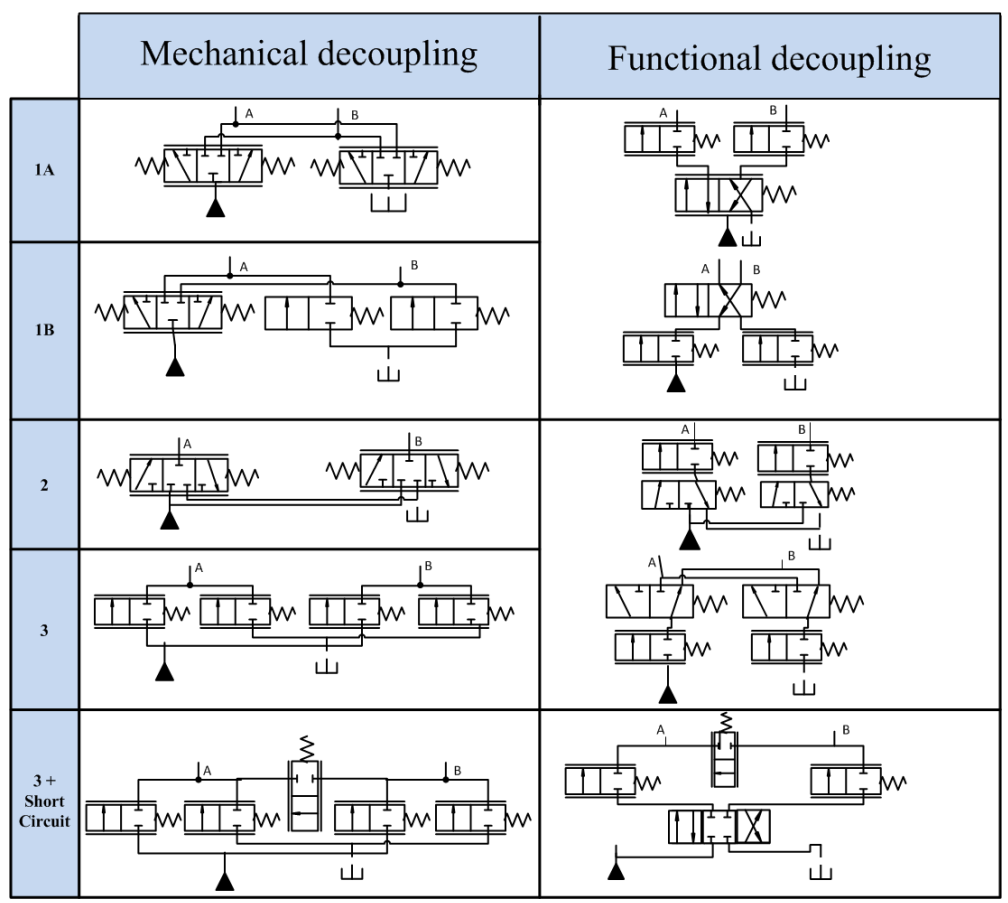

Figure 8 The main decoupling types that are used for Independent metering configuration [5].

developed by Caterpillar [80] and [81]. A programmable valve is a term represents a configuration of a five electronically controlled poppet valve 82 . These valves' performance was evaluated by [83 and [77] and, their model was developed by [84. After the manufacturing process for these valves, a deviation in performance can be noticed which affect the IM system overall and to overcome this a calibration algorithm can be used [85]. A novel auto-calibration statetrajectory control method for IM uses a four poppet valve configuration or a Wheatstone bridge [18. Inserting electronics and sensors to IM valves improves the controllability and overall system performance. HUSCO's INCOVA developed a brand of this configuration [86. Figure 10 shows the configuration of twin-spool valve architecture. Using electronics and sensors in these hydraulic systems increases the failure due to the harsh environment, and to overcome this drawback, EATON improved a failure operational control algorithm [87]. 
Regarding digital hydraulic approach using DFCU. Different kinds of valves are used for digital hydraulics. These are bi-stable on/off valves improved by Uusitalo, a monostable needle improved by Karvonen and a wide array of digital hydraulic systems improved by Bucher Hydraulics [88. These valves arrangement can be used for IM as simulated by [73]. Table 22 includes the IM valves that have been produced to implement the independent metering system in the mobile machines.

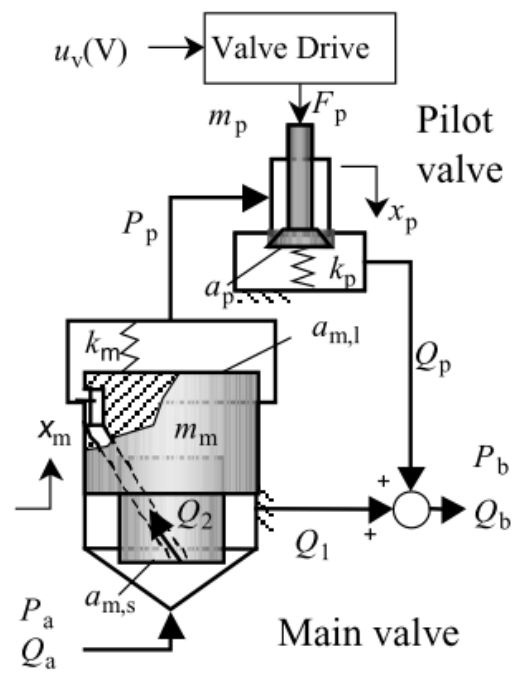

Figure 9 The main schematic of electrohydraulic poppet valve where $Q_{p}$ is the pilot flow, $m_{m}$ is the main poppet, $P_{P}$ is the pilot pressure, $P_{a}$ is the main pressure source, $Q_{2}$ is the feedback control flow, $Q_{b}$ is the total flow. [83] 


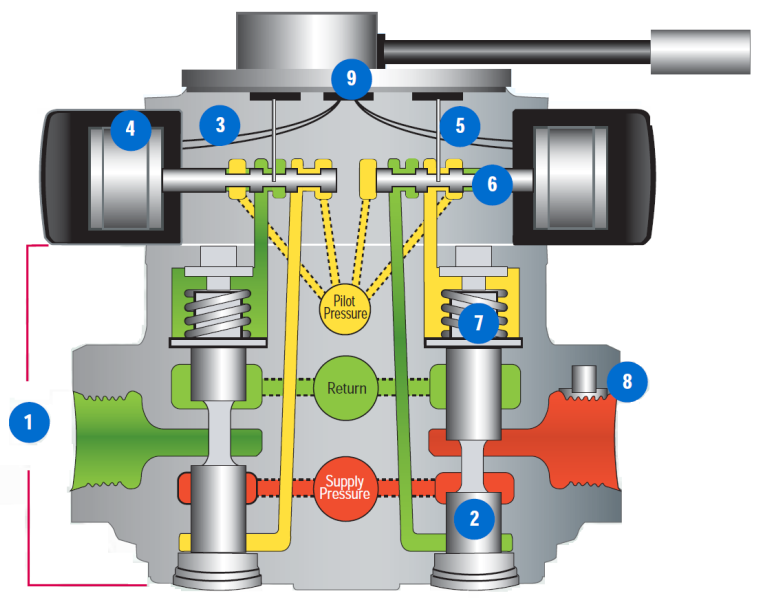

Figure 10 The Eatons Ultronics twin independent spool valve (EATON 2010). 1 main stage valve block, 2 - independent spool for metering, 3 - pilot valve, 4 - low power voice coil actuator, 5 - centring spring, 6 - pilot spool, 7 - position sensor, 8 thin film pressure sensor, 9 - embedded micro electronics

Table 2 Details of the industrially produced independent metering valves .

\begin{tabular}{|l|l|l|l|l|l|}
\hline Manufacturer & Product & Flow Rate & Hysteresis & $\begin{array}{l}\text { Response } \\
\text { Time }\end{array}$ & $\begin{array}{l}\text { Pressure } \\
\text { Drop }\end{array}$ \\
\hline EATON & $\begin{array}{l}\text { EPV10 } \\
\text { 89]. }\end{array}$ & $30 \mathrm{~L} / \mathrm{min}$ & $<4 \%$ & $35 \mathrm{~ms}$ & $200 \mathrm{bar}$ \\
\hline BUCHER & WS22GD[90]. & $30 \mathrm{~L} / \mathrm{min}$ & $<5 \%$ & $20 \mathrm{~ms}$ & $350 \mathrm{bar}$ \\
\hline EATON & $\begin{array}{l}\text { CMA90 } \\
\text { 91]. }\end{array}$ & $90 \mathrm{~L} / \mathrm{min}$ & $\begin{array}{l}\text { sub- } \\
\text { micron }\end{array}$ & $24 \mathrm{~ms}$ & $25 \mathrm{bar}$ \\
\hline EATON & $\begin{array}{l}\text { CMA200 } \\
\text { 92]. }\end{array}$ & $200 \mathrm{~L} / \mathrm{min}$ & $\begin{array}{l}\text { sub- } \\
\text { micron }\end{array}$ & $24 \mathrm{~ms}$ & $35 \mathrm{bar}$ \\
\hline HUSCO & EHPV[93]. & $\begin{array}{l}75,150,800 \\
\mathrm{~L} / \mathrm{min}\end{array}$ & very Low & $100 \mathrm{~ms}$ & $15 \mathrm{bar}$ \\
\hline
\end{tabular}

However, the major characteristics of the valves that have been studied to implement the IM are included in Table (3), as obtained from [4. A significant recent development in the IM valves is developing the new stepped rotary flow control valve [94, 95, 96]. It has been developed to replace the poppet, valvisotr, valve and to overcome some of its shortcomings such as flow accuracy and stability. Embedding the stepped rotary valve into the IM configuration allows implementing an advanced intelligent control system and improves the system 
performance by avoiding depending on the fluid as part of the control system which is a major drawback of the valvistor valve. The stepped rotary valve is shown in Figure [11. It has two main parts which are the orifice and the stepper motor. A sensor-less activation algorithm for the rotary valve has been developed in [97. This technique increases the controllability and reduces the fault rate by excluding rotation feedback sensor.

Table 3 Different flow control valves and their characteristics

\begin{tabular}{|c|c|c|c|}
\hline Characteristic & Spool & Poppet & Digital \\
\hline Flexibility & Low & Medium & High \\
\hline Flow accuracy & High & Medium & Low \\
\hline Redundancy & Low & Medium & High \\
\hline Manufacturing cost & High & Medium & Low \\
\hline Anti-leakage & Low & Medium & High \\
\hline Sensitive to contamination & High & Medium & Low \\
\hline Stability & High & Medium & medium \\
\hline
\end{tabular}

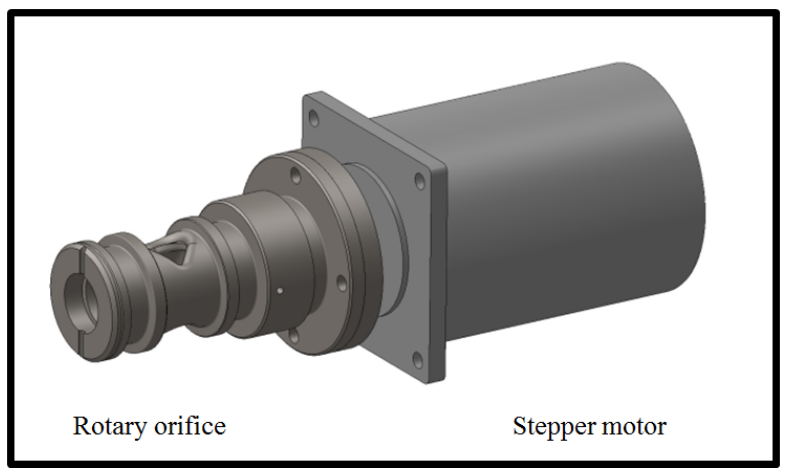

Figure 11 The main schematic of electrohydraulic rotary flow control valve [95.

\section{IM control systems}

Inserting software control as a main part of hydraulic system grants implementing intelligent control techniques. The hydraulic programmable control system contains three main levels which are illustrated in Figure 12 47. The upper level is the mode switching level which allows energy regeneration and 
recuperation. It performs the mode switching to select the most efficient mode which allows energy regeneration and recuperation. The selecting technique relies on the system's status such as pressure and velocity. In the lower level, the selected valves in each mode are activated to produce a flow rate which controls the cylinder speed. The primary level is to control the pump pressure and flow 4. The review in the paper focuses on the upper and lower levels.

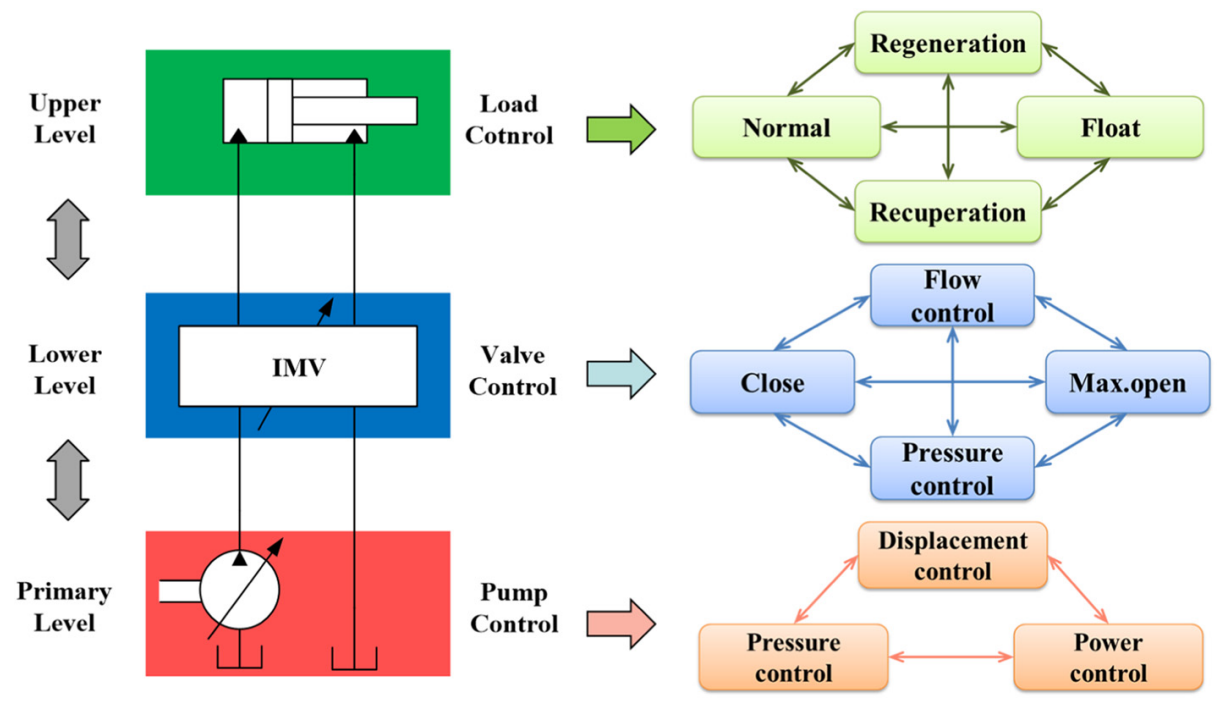

Figure 12 The main three control levels of programmable hydraulic control system 4.

For the lower level, different control parameters can be used for independent metering as shown in Figure 13 . These parameters can be separated into flow, pressure difference and displacement control. As the flow and displacement controls rely on electronic sensors, they do not exist in hydro-mechanical types that use pure mechanical components.

As the separation of actuator metering increased the degree of freedom, different control strategies can be applied and investigated on the system. Three main control systems can be implemented on IM as shown in Figure 145 .

The first approach, which is Feed-forward control, is mainly used in mobile machinery, and the operator who closes the loop [5] and [77. The second 


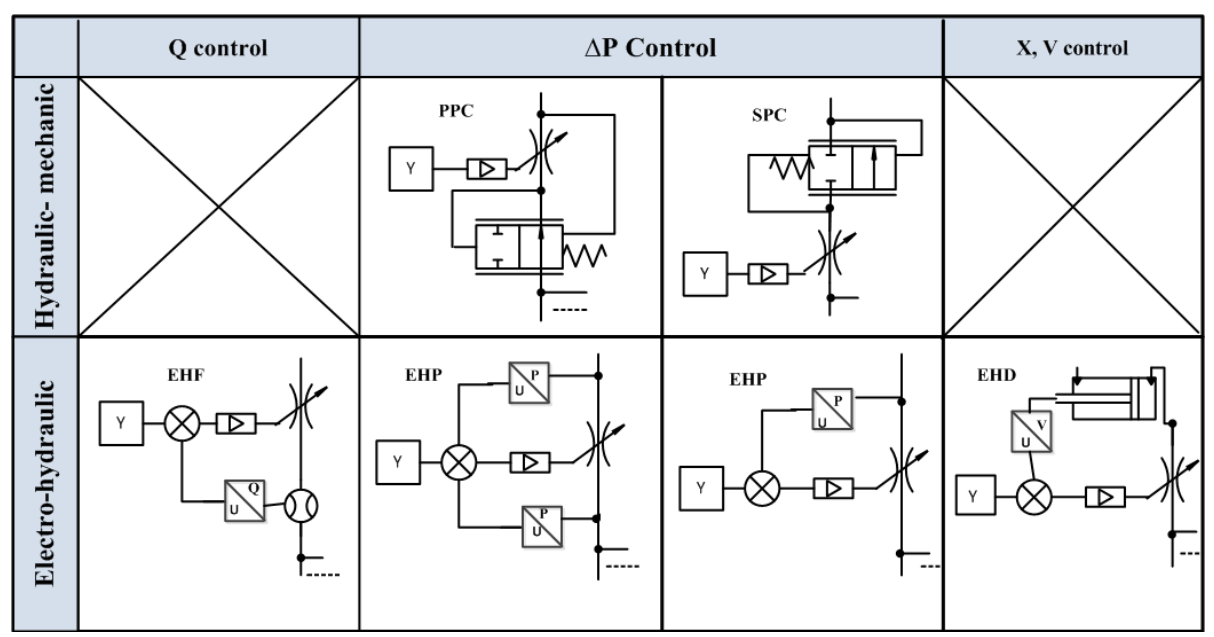

Figure 13 Different control parameters implemented for IM where PPC-Primary pressure compensator, SPC-secondary pressure compensator, EH-Electrohydraulic, PPressure, F-Flow, and D-displacement [5].

type is closed loop control feedback, Single Input Single Output (SISO), to ensure that the output follows the trajectory command. The last one, which is the Multiple Input Multiple Output (MIMO), is a closed loop control system. It is used to control more than one target variable where different states are controlled at the same time in IM. These states are coupled together. The decoupling between them can be performed using MIMO control. A study for MIMO control approaches and the pressure compensator effect was conducted by [16. Different iterations were performed to decouple these factors. Decoupling between the actuator velocity and pressure using a combined pump and valve control [98. The aim of the study in [17] was to decouple the response and the pressure level in the hydraulic actuator using four orifices. Some researchers designed decoupling between the velocity and pressure in the hydraulic actuator and this requires velocity feedback [17. This control approach achieved a good decoupling, but this depends on the quality of the velocity feedback signal. A $H_{\infty}$ loop-shaping approach without taking measures to dampen the system was investigated [36]. Conversely, the pressure compensator is an important component reducing cross-talking or the coupling between two valves [16] and [77. 


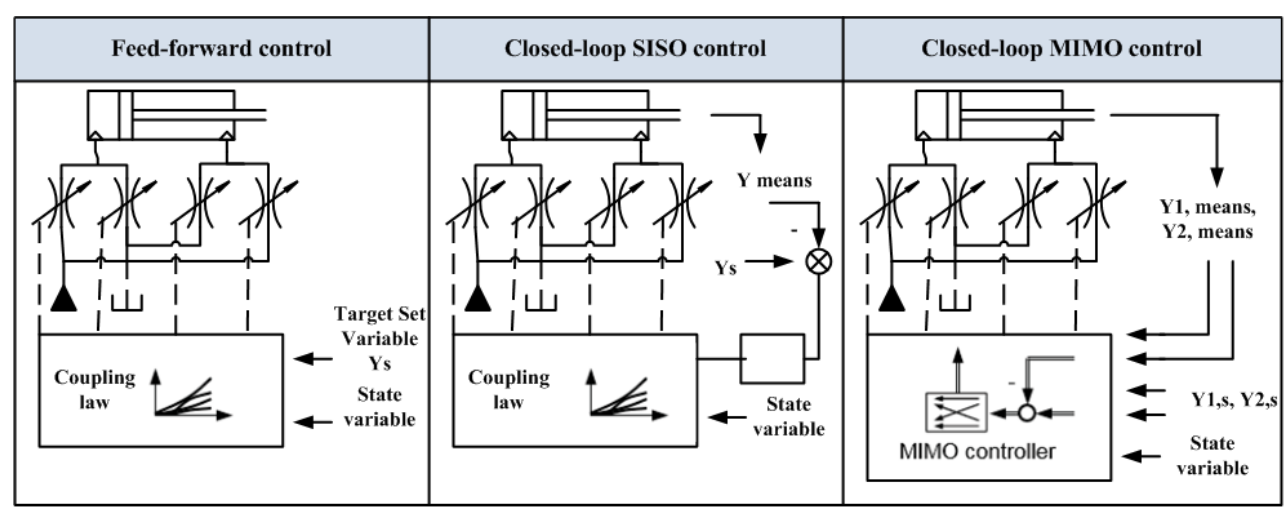

Figure 14 Different control approaches for Independent Metering [5].

Moreover, a flatness-based control algorithm was used to allow manipulation of the cylinder speed and the pressure level separately. It was used in the inner layer for the continuous mode switching technique within Modicieny approach [99] and [100]. On the other hand, load oscillation appears during movement using separate meter-in separate-meter out (SMISMO) system, precisely when the load stopping after moving. An optimal approach based on Hamiltonian method was developed in [101].

Regarding single loop controllers, PID controllers are widely used to activate valves with their flow maps. The Fuzzy PID controller was improved to enhance the dynamic performance of two stages servo valves to form independent metering system 102. Also PID controllers are used to activate variable displacement pumps [47. It was used for velocity and pressure control for actuators in multifunction systems 103 and 104. It was also used to improve performance for a hydraulic excavator [105].

Adaptive control is an approach that changes the controller in real time. This maintains the desired level of a control system especially when the parameters of the model are uncertain or nonlinear as in hydraulic systems [106]. Adaptive control was used for five valves scheme [107] and [25]. Usually, IM is configured using four valves which is called Wheatstone bridge. Additional valve enables precise control of direct cross port flow. This control technique was in- 
cluded in an improved hybrid system using a three-level control system and an accumulator [26. Moreover, indirect adaptive robust dynamic surface control (IARDSC) method was developed to enhance the performance of IM system by reducing the internal uncertainties and external disturbances using (IARC), while the dynamic surface control (DSC) is used to deal with the inherited explosion of term [108. As the IARC is good for constant parameters estimation, it's performance is poor when the is fast parameters changing. The effect of fast parameters change produces an explosion of the term and is used to overcome it 109. Vibration is one of the drawbacks of the IM method due to the lack of damping on meter-out. A hybrid control method combining dynamic pressure feedback and active damping controller was designed, and pole-zero assignment approach was implemented to capture the optimal damping under large variation of operating conditions [110].

\section{IM operation modes}

For the high level of the programmable hydraulic, the independent metering structure allows for different operating modes which reduce power consumption. These operation modes represent certain fluid paths into and out of the actuator. Also, they are variable due to load changes and supplied pressure. Some of these modes such as regeneration were not achievable using $4 / 3$ valves [111. The main scheme used by Shenouda in [111] and Sameh in [112 to study an independent metering system was the Wheatstone bridge as shown in Figure 15.

This configuration allows for five modes which are as follows:

1. Power extension mode (PE).

2. Power retraction mode (PR).

3. High side regeneration extension mode (HSRE).

4. Low side regeneration extension mode (LSRE).

5. Low side regeneration retraction Mode (LSRR). 


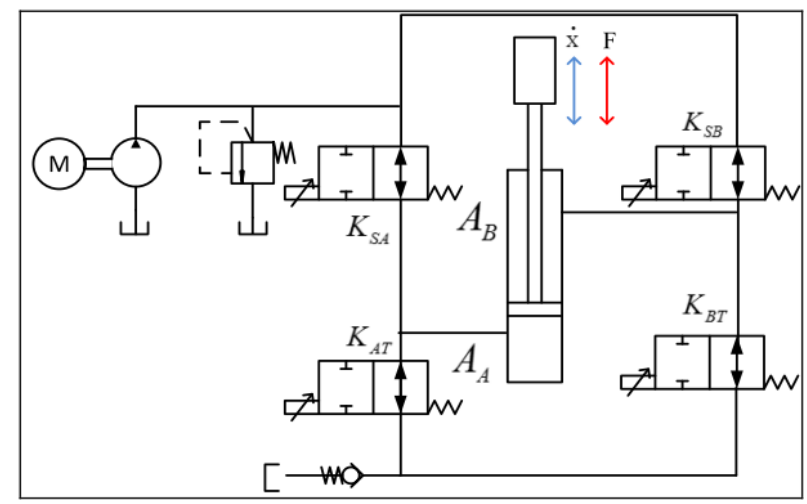

Figure 15 The main IM diagram used by Shenouda 28 where $K_{s a}$ is the valve between the pump line and the cylinder head chamber, $K_{a t}$ is the valve between the tank line and the cylinder head chamber, $K_{s b}$ is the valve between the pump line and the cylinder rod chamber, and $K_{b t}$ is the valve between the pump line and the cylinder head chamber.

Power extension mode is performed by supplying the fluid from the pump to the actuator head chamber using inlet port, while the fluid drained from the actuator to the tank using the outlet branch. The next operation mode is power retraction. Its the opposite of the power extension mode. These two modes are illustrated in Figure 16. And they are the most energy-consumable modes.

Regeneration modes are separated into high side and low side. The High Side Regeneration Extension Mode (HSR) is shown in Figure 16. The high side

regeneration is achieved when the fluid is passed from the rod chamber to the head chamber using the high connection point of the bridge. The recirculated flow is not enough, so the difference supplied by the pump itself. This indicates that the power extension mode provides force more than high side regeneration extension while HSRE can achieve more speed than power extension 28.

The low side regeneration appears when fluid regeneration is performed at low point connection. Low side regeneration has two types which are low side regeneration retraction mode and low side regeneration extension mode. These modes are shown in Figure 16. The latter happens when the load is lowering and the gravity helps. The first happens when the load is lowering using its gravity and the outlet fluid is fed into the head chamber. 


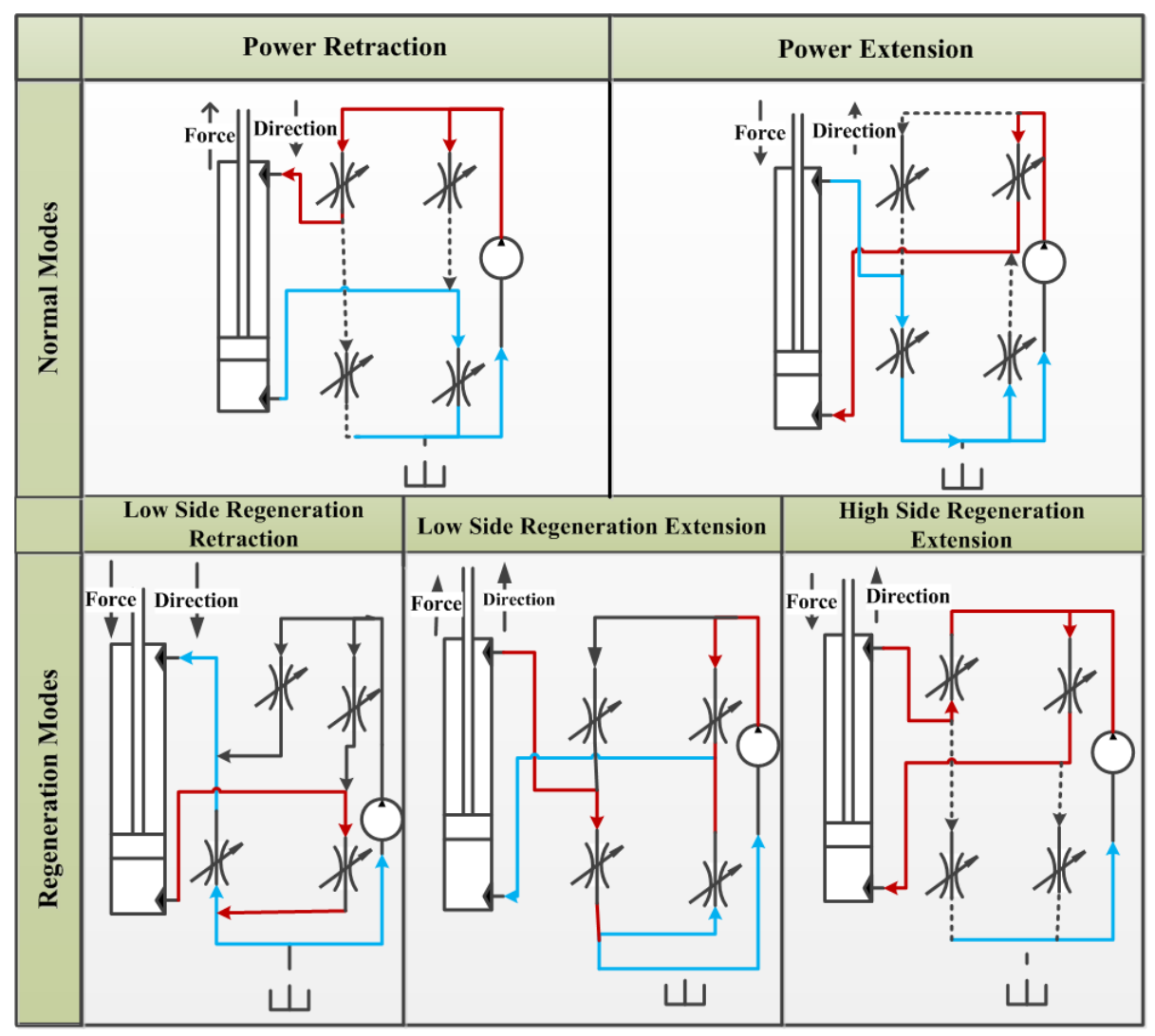

Figure 16 The five operation modes of the independent metering

As shown in Figure 17, different terms for the operation modes were defined based on the meter-in, meter-out, and the pump activation[77]. These terms are as follows:

1. Recuperative mode: The energy is gathered only from the load to actuate actuators such as pumps and motors.

2. Neutral: No energy is needed to perform the operation.

3. Regenerative: When the lower flow is required from the pump. Flow is mainly obtained from the actuator with a high load.

4. Normal mode: These modes when all the flow is obtained from the pump. 


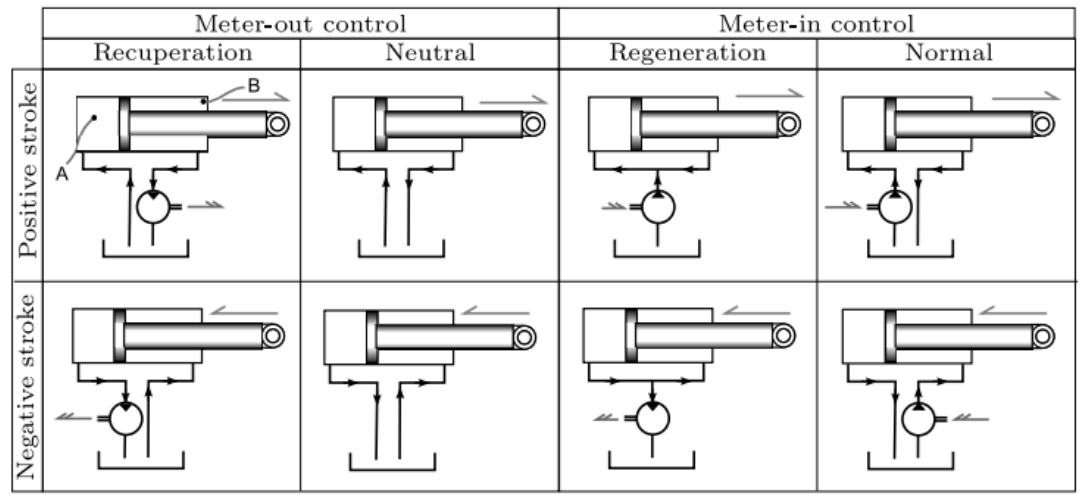

Figure 17 Main operation modes as indicated by 77

There are other terms for the modes in Figure 17] as indicated in 99. The terms of the Normal mode, Neutral mode, Regeneration mode, and Recuperation mode, respectively, are the Normal mode, the Low-Pressure Regeneration mode, the High-Pressure Regeneration mode, and the Reverse mode. Figure 18 shows valves activation in every control mode based on a five valves architecture. These modes, with their valves, are used to implement Modiciency technique which is discussed in 99.

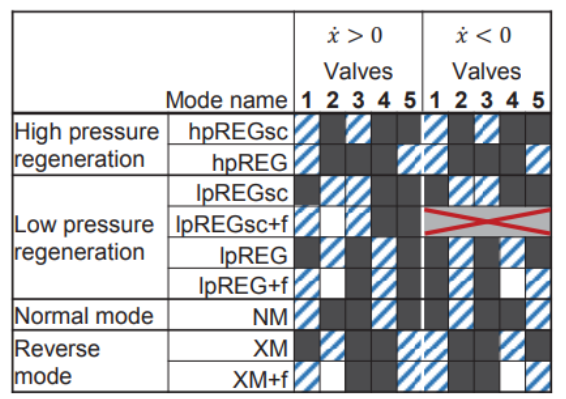

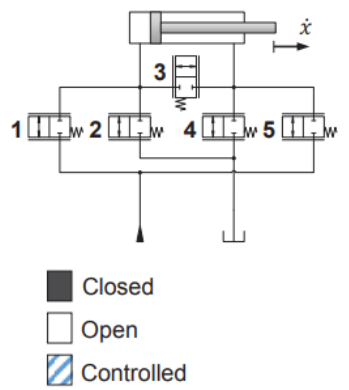

Figure 18 Valve architecture for Modiciency technique. The hpREG is for the high pressure regeneration mode, lpREG is for the low pressure regeneration mode, $\mathrm{Nm}$ is the normal mode, the Xm is the reverse mode, the suffix (sc) is for the short circuit, and the suffix (f) is for additional pressure obtained from the supply pressure line 99

STEAM systems introduced a different division based on operating states. The operation state refers to each discrete valve setting while the operating mode is a composition of the state and the load [75. Figure 19 shows the discrete 
operating states for STEAM systems using low, medium and high-pressure lines.

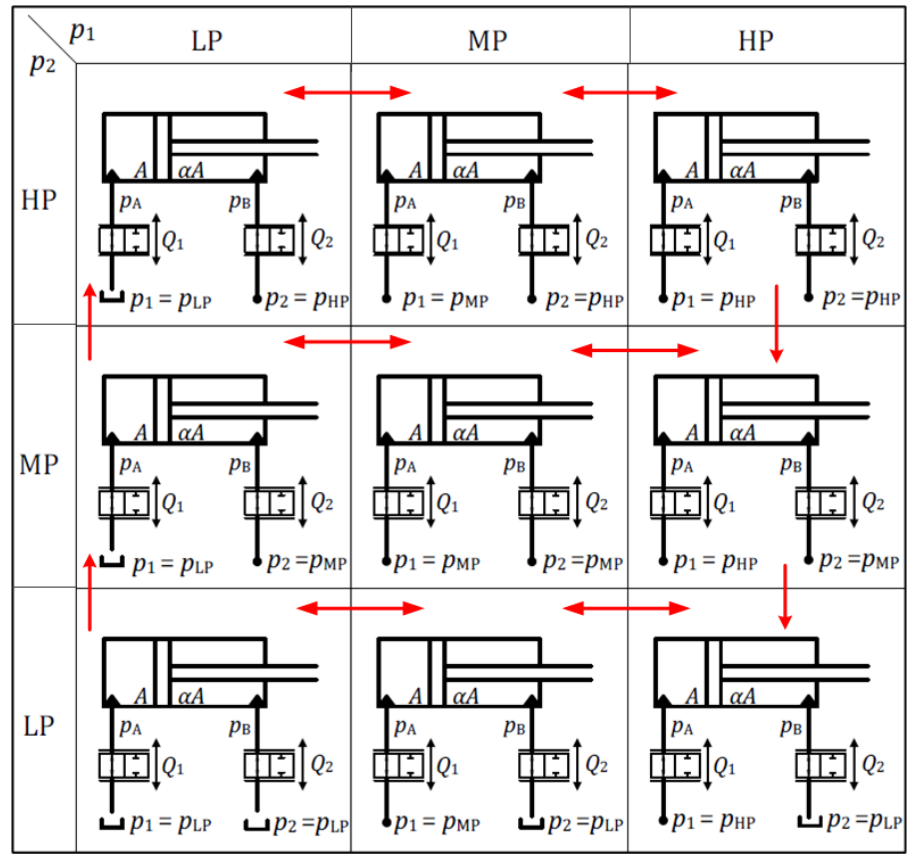

Figure 19 STEAM system operation modes where P: pressure, L: low, H:high, M: medium and $\alpha$ : ratio 75 ]

In IM, operation modes are separated into four quadrants as shown in Figure 20 (a) and their power division in (b), 46].

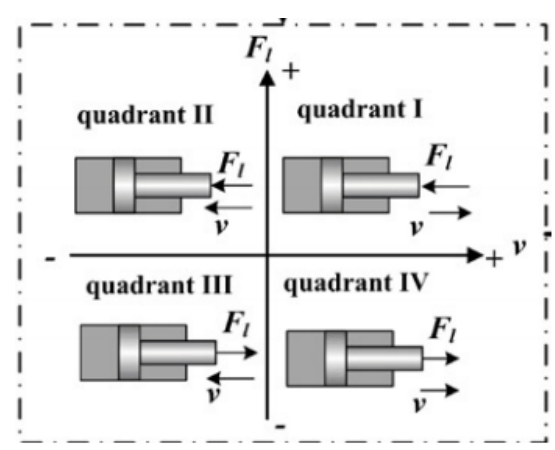

(a) Operation mode based on quadrants divisions

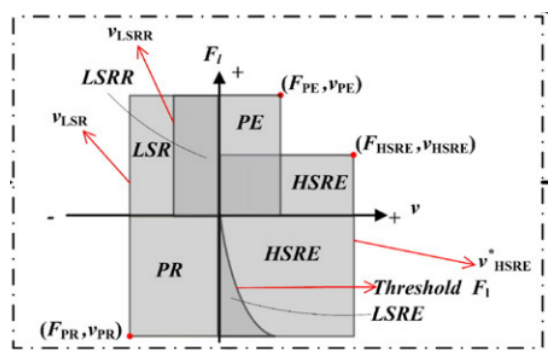

(b) Limits for the modes division

Figure 20 Limitation for the operation modes in IM 
These quadrants are separated as follows:

1. This quadrant has two operation modes which are power extension and high side regeneration retraction mode. The limits for PE are implemented by Eq. (1), and for HSRE by Eq. (2).

$$
\begin{gathered}
{\left[F_{P E}, V_{P E}\right]=\left[P_{s, \text { max }} \cdot A_{a}, \frac{q_{s, \text { max }}}{A_{a}}\right]} \\
{\left[F_{H S R E}, V_{H S R E}\right]=\left[P_{s, \text { max }} .\left(A_{a}-A_{b}\right), \frac{q_{s, \text { max }}}{\left(A_{a}-A_{b}\right)}\right]}
\end{gathered}
$$

2. This has two modes which are the low side regeneration retraction Eq (3) and Eq (4) when the velocity exceeds the limits of LSRR mode.

$$
\begin{gathered}
{\left[F_{L S R R}, V_{L S R R}\right]=\left[F_{l}, \frac{q_{L S R R}}{A_{b}}\right]} \\
{\left[F_{L S R}, V_{L S R}\right]=\left[F_{l}, \frac{q_{s, \max }}{A_{b}}\right]}
\end{gathered}
$$

3. Power retraction mode PR

$$
\left[F_{P R}, V_{P R}\right]=\left[P_{s, \max } \cdot A_{b}, \frac{q_{s, \max }}{A_{b}}\right]
$$

4. Low side regeneration extention(LSRE)

$$
\left[F_{L S R E}, V_{L S R E}\right]=\left[F_{l} \cdot A_{b}, \min \left(\frac{q_{L S R E 1}}{A_{a}}, \frac{q_{L S R E 2}}{A_{b}}\right)\right]
$$

Shenouda designed three valve modulation modes. These modes are continuous and they include the five discrete modes [28. They are the powered high side regeneration extension mode (PHSRE), the powered low side regeneration retraction mode (PLSRR) and the powered low side regeneration extension mode (PLSRE). As indicated in the same source, this method can achieve smoother velocity control and higher force capability compared to discrete modes. 
Regarding STEAM systems, division is based on states as illustrated previously in Figure 19. These divisions could be regeneration, recuperation, resistive float or assistive float. It is normally difficult to apply all these divisions and this is based on the actuator type and dimensions [43].

\section{Mode switching methods}

Mode switching should occur when the mode capability is no longer sufficient. A suitable mode switching is used to minimise losses and decrease switching problems which are switching instability and unsmooth switching [46]. Many algorithms were developed for mode switching. As these modes directly affect the performance of the actuator, smooth and fast switching is crucial. A selection method based on cylinder force, velocity and required force using an ACR controller was improved [13] and [25]. A mode switching using a fitness function was implemented [11]. This function receives problem parameters as inputs and suggested the solution to get out the optimum solution. It should be able to utilise fast computing speed and quantitatively measure the suggested solutions. Also, the continuous mode switching using three valves simultaneously was discussed [46]. A Mode transition, based on meter-in or meter-out control, was developed as shown in Figure 21 [77. Meter-out control uses a meter-out valve to control the speed, has two operation modes which are recuperation mode and a neutral mode. Changing between these two modes depends if the load is higher than the pump pressure. When neither of these operation cases can be used, the system will start using oil from the pump. This is called meter-in control which represents the right part of Figure 21

Moreover, mode switching technique for the STEAM system which relies on two pressure lines [113] and [114]. This technique is based on a finite state machine. The main idea is to split the modes into three groups, namely $Q_{H}, Q_{M}$, and $Q_{l}$. Each group contains three states, for instance $Q_{M}$ includes $Q_{M P, H P}$, $Q_{M P, M P}$, and $Q_{M P, L P}$. The principle of the mode switching is to keep the pressure in one chamber and change it in the other to prevent interference 
Too low pressure drop over

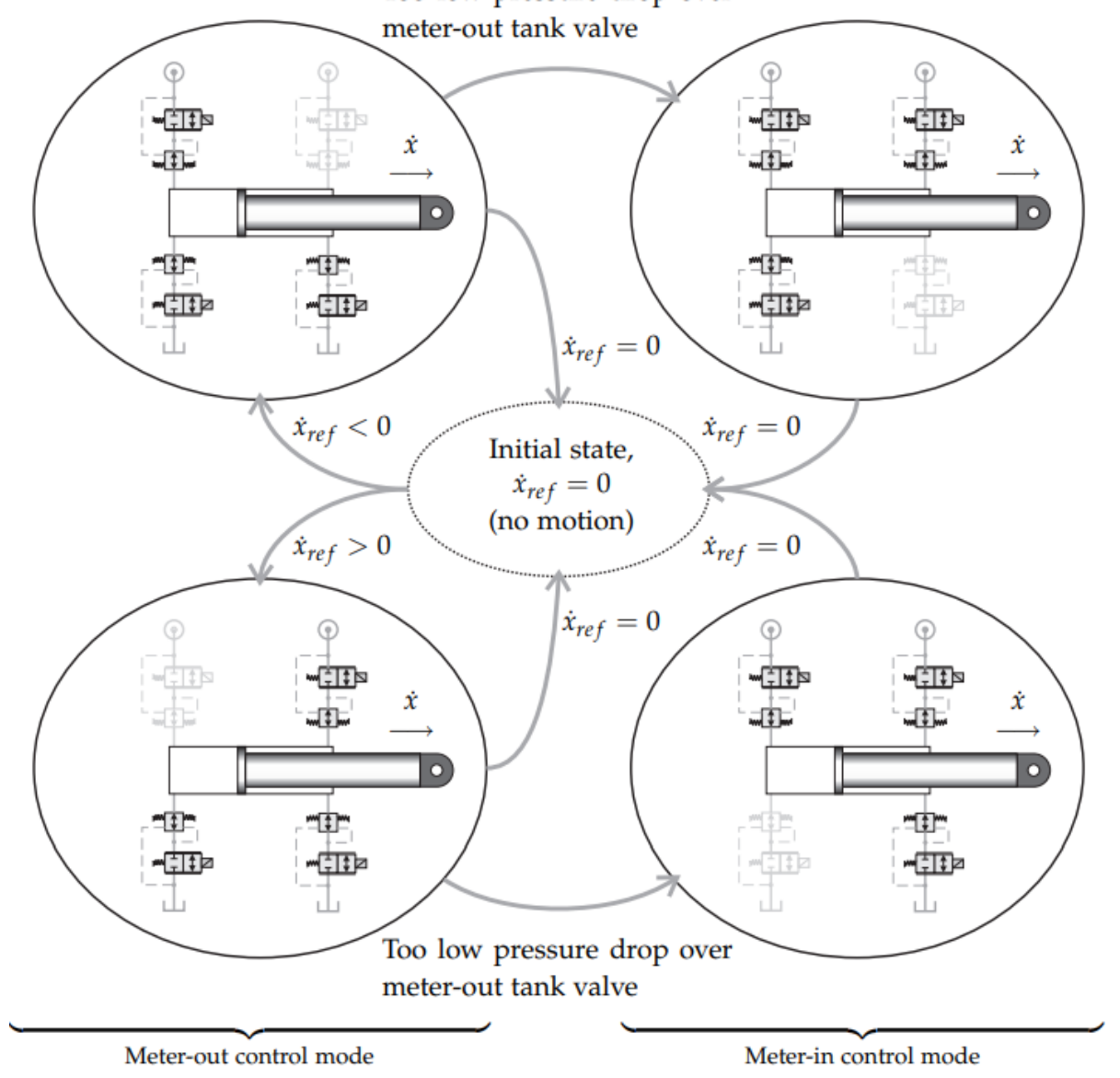

Figure 21 Mode switching division for four valves IM configuration [77]

between set of states in the same group as shown in Figure 19 For example, in

the group $Q_{M}$, the head chamber valve was fixed on pressure line $M P$ during changes and the transition was for the rod chamber pressure between $H P, L P$ and $M P$ according to the state power capability. The change between $Q_{H}$, $Q_{M}$, and $Q_{T}$ was performed by fixing the rod chamber valve and adjust the head chamber valve.

On the other hand, the technique used for switching in the Modiciency approach is based on controlling the velocity and pressure level using the MIMO control. This technique allows for the continuous mode switching based on 
modes illustrated in Figure 18. The continuous mode switching is shown in Figure 22 .

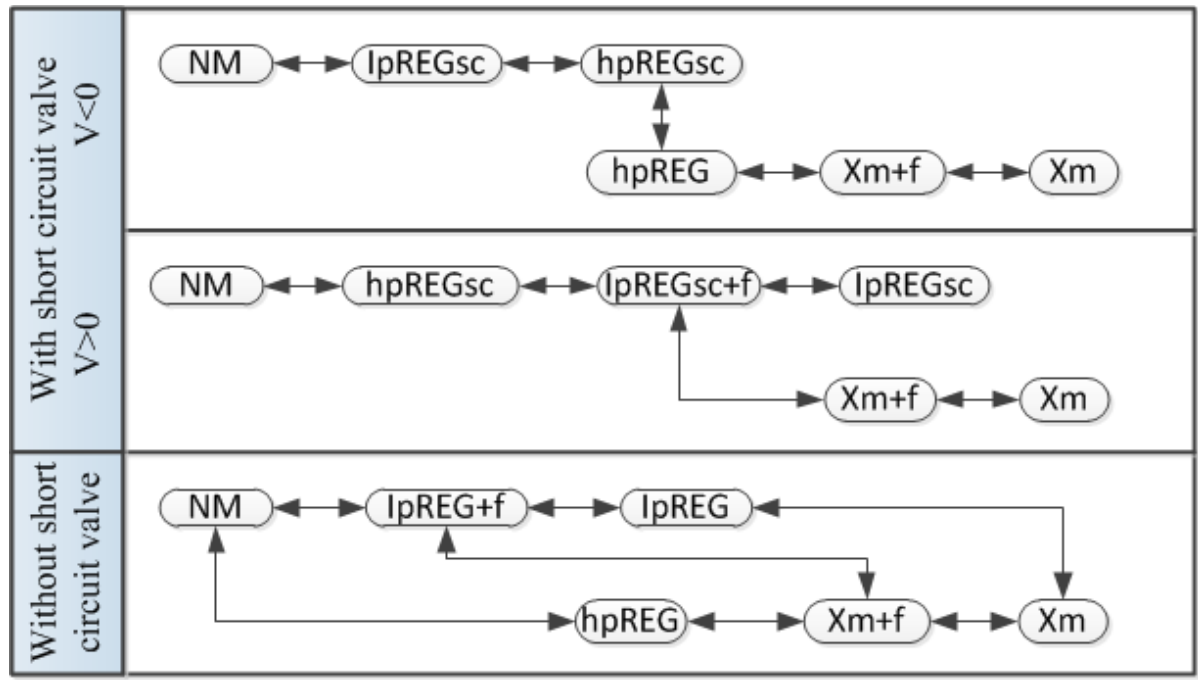

Figure 22 The modes switching for the Modiciency technique 77 .

A bumpless mode switching algorithm is used to overcome discrete switching problems [46. This algorithm contains two parts which are dynamic dwell-time and bidirectional latent tracking loop. The dynamic dwell-time is used to reduce transient instability by slowing the transformation into sufficient time. The time value is obtained based on the Multi-Lyapunov function. The bidirectional latent tracking aims to solve the unsmooth switching by eliminating the discontinuity of control signals. With regard to digital valve systems that can be used to implement independent metering, Linjama has improved a stepwise change in valve states [115].

\section{IM layouts}

In general, the combination between the hydraulic circuits and the IM has different forms as follows:

1. The combination between the traditional hydro-mechanical load sensing 
and the IM. In this configuration, the pump is driven hydro-mechanically and the poor damping is one the main system shortcomings.

2. The combination between the electronics load sensing system and the IM valve configuration. The electronics load sensing relies on Electronics Controlled Pump (ECP) to control the circuit pressure and flow.

3. Combine the closed circuit with IM to eliminate the throttle losses [116. The drawback of this configuration is a functional failure under overload condition [117. So, ECP open circuit combining IM was introduced by Kim in 118.

4. The last configuration which includes inserting accumulator as an energy storage unit that allows reusing the fluid during operation [26].

Tabor designed a control system configuration for four valves independent metering as shown in Figure 23 The control system is based on a microprocessor that coordinates the work between different control portions which are a pressure controller, a function controller and a system controller [119]. The function controller reads the pressure from the meter-in, meter-out, source pressure, and the tank pressure. The system controller is responsible for reading the pressure and speed commands, then it selects the operation modes and identifies the required flow rates for each valve (21-24) in Figure22. Also, the cavitation is observed during operation as if it appears the controller loses its functionality. In the end, the pressure controller is used to control the pressure from the pump and tank in order to save energy and prevent cavitation. The practical implementation of a similar IM valve control system combined with pump control algorithm was performed on a 20-ton excavator by Hyundai Heavy Industries Co., Ltd [120] and indicated a saving of energy of up to $10 \%$ comparing to conventional excavator which rely on normal spool valves. 


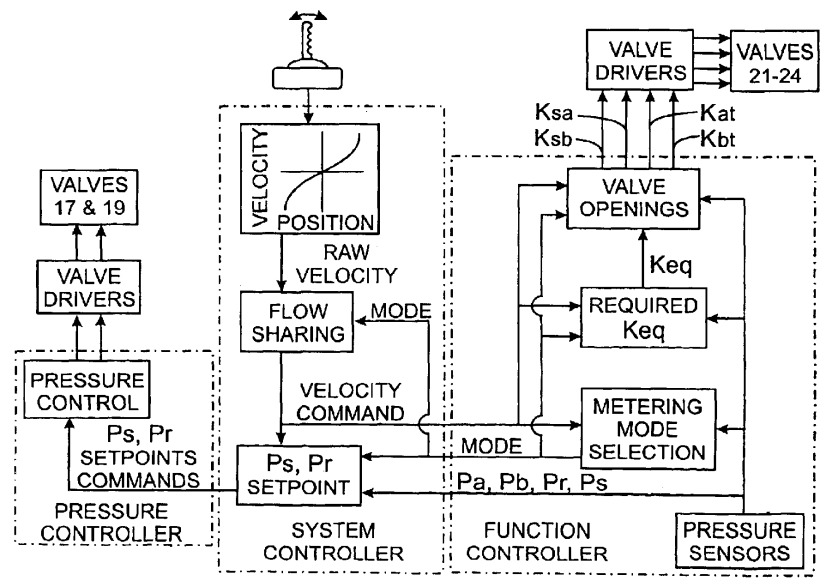

Figure 23 Tabor control scheme for four valves IM [119]

A novel structure for mobile machines with the common pressure compen435 sator was developed using the minimum number of sensors and simple control algorithms [11. It was developed to be more acceptable to industry, as illustrated in Figure 24. Its main strategy is controlling the velocity from the meter-in using open loop approach. The meter-out is controlled using closedloop feedback. To overcome the nonlinear relationship between the meter-out signal and the pressure compensator throttling which is inherent in the system due to the closed-loop feedback, the meter-out value should rely on the head chamber pressure which can be determined from the pressure drop value on the pressure compensator. 


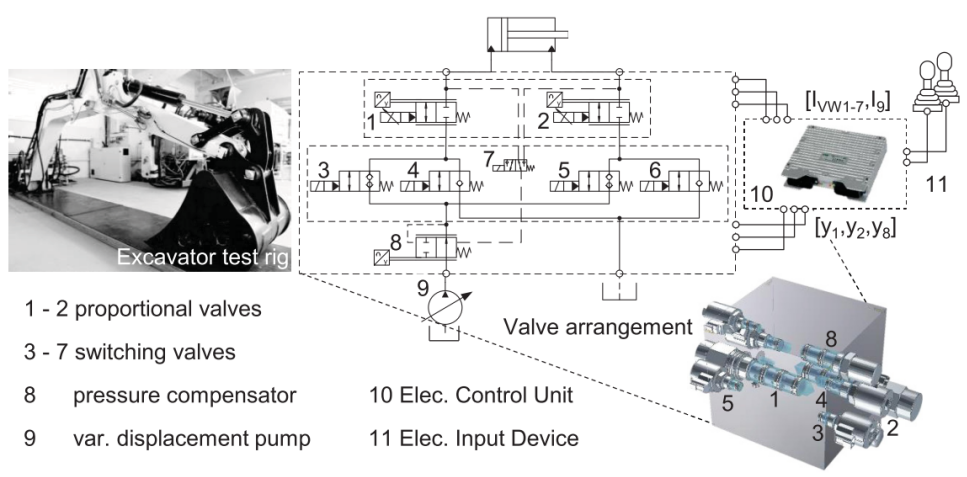

Figure 24 Novel pressure compensated control scheme [1]

For a hybrid system, a three-level control system was designed [26]. This control system aims to cooperate the operations between the main pressure source, the accumulator and actuators to reduce the power consumption and increase controllability using adaptive control. For STEAM systems, RWTH Aachen university improved a control system using two proportional 2/2 way valves and six switch valves for each actuator [31. This system is shown in

(a)
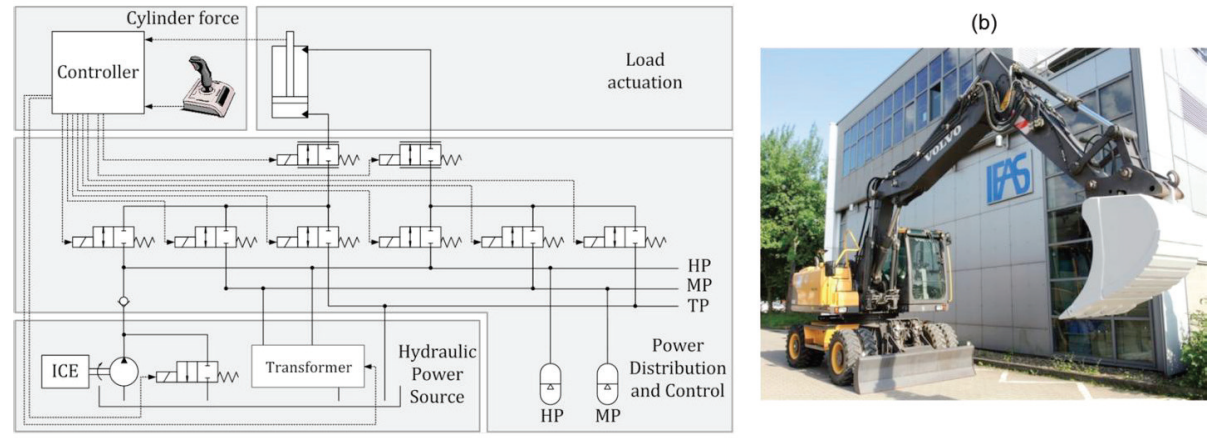

Figure 25 STEAM system configuration for hydraulic excavator 31

a new system that able to adapt to load fluctuation was designed [57]. Every actuator contains two $3 / 2$ switching valves and one proportional valve. The proportional valve is to control the cylinder movement while the switch valves are used to connect the proportional valve to the pressure lines as shown in 
Figure 26. The oscillation due to the pressure line switching can be reduced by a step change of the switching valves.

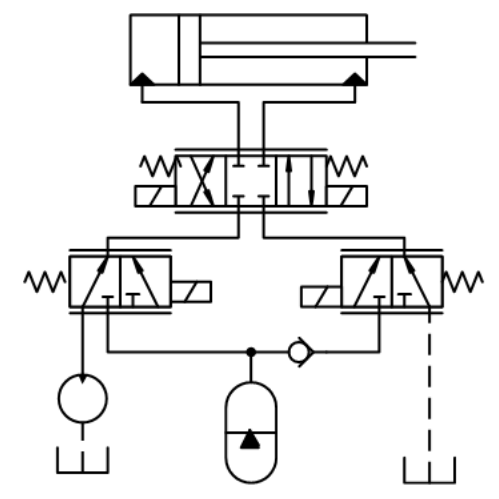

Figure 26 Valve control concept with intermediate pressure line [57]

A meter-out control with pressure compensator was improved [20]. This system, which is illustrated in Fig. 28, was an improvement because most of the manufacturers do not offer bidirectional poppet valves.

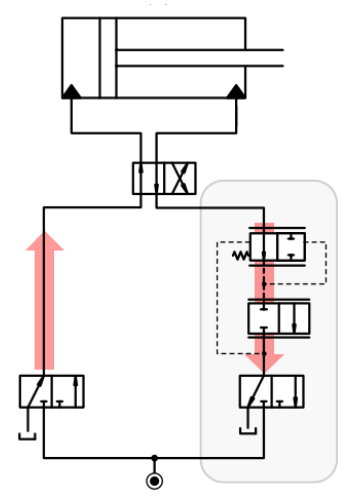

Figure 27 Meter-out control with the pressure compensator 20]

A flow on demand concept which was developed to reduce the losses in load sensing systems relies on the joystick commands or the valve position in contrast to LS that depends on the highest consumer pressure [65] and 121. Combining flow on demand concept with independent metering leads to better efficiency and controllability [122]. As illustrated in Figure 28, the controller receives 

control the variable displacement valve. The accumulator is used to allow energy regeneration.

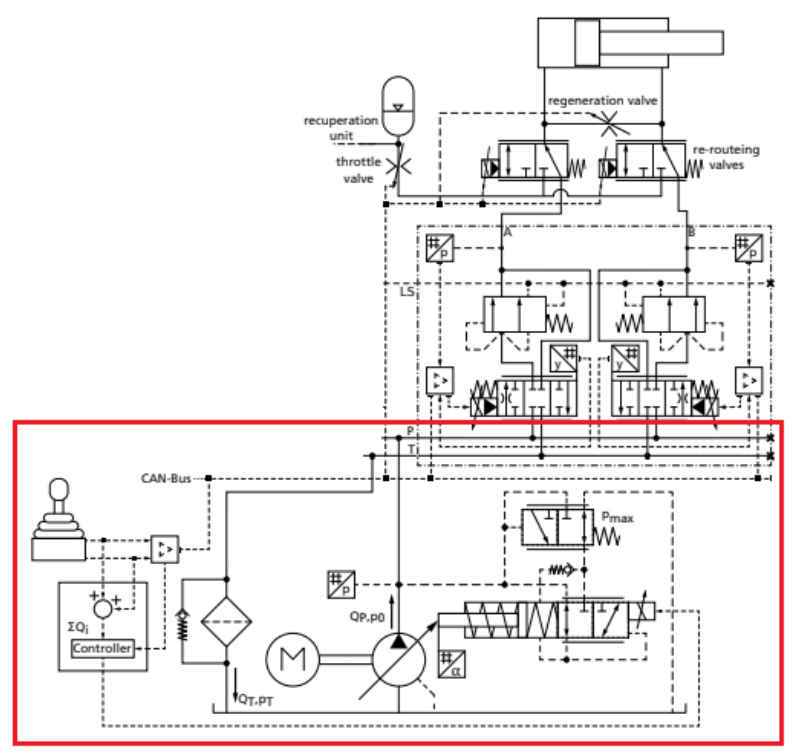

Figure 28 Flow on demand circuit combined with IM system, the highlighted part represents the flow on demand circuit 122 .

An example of load sensing combined with pressure compensation and independent metering is shown in Figure 29. This configuration grants two pressure compensation methods which are the meter-in pressure compensation (MIPC) and the meter-out pressure compensation (MOPC). The pressure reducing valve is used between the metering valves block and the LS part to reduce the required pressure from the pump as minimum as possible and this relies on the pressure sensors 1 and 2. The feedback pressure 3 and 4 values are used to form a closed 475 loop control signal for the controller 2. 


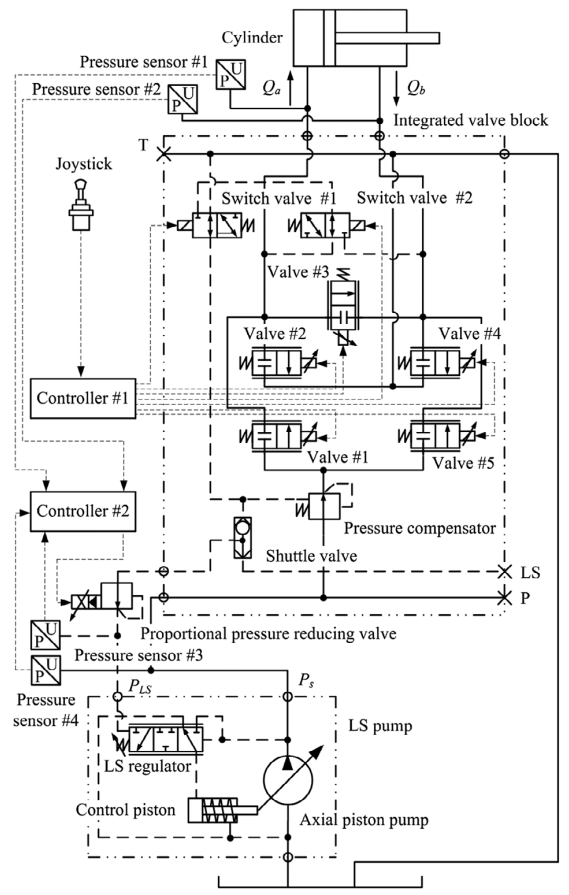

Figure 29 Hydro-mechanical pressure compensated load sensing circuit with independent metering configuration. 67.

The independent metering has been used to drive motors in hydraulic mobile machines. For example, IM is used to drive the swing part of a hydraulic excavator similar to the driving technique that was developed in [123, 52. The study showed that IM improved the dynamics performance of the excavator swing by controlling the throttle orifice size. A control algorithm was developed by Caterpiller to control the swing motor in order to save extra energy [124]. Also, an accumulator was attached to the swing motor to allow energy regeneration as investigated in 125. A flow matching technique for hydraulic excavator swing was developed in 126 . This technique reduces the energy losses by producing the required flow by the motor, and improves the system dynamics by a separate control of the throttles for a high inertia component.

A novel hydromechatronics configuration of the independent metering using a stepped rotary flow control valve has been developed. This configuration, 
shown in Figure (30), is termed as Micro-Independent Metering (MIM) due to the activation technique of the valve [127. A new control algorithm has been developed for the MIM systen. It grants the user extra options such as smoothness activation which changes the step division of the stepper motor. Moreover, this configuration transfers the traditional control, using the valvistor valve, from infinite positioning to finite steps. From the controllability side, the new system increases the stability margin by eliminating the effect of the fluid disturbances on the control elements which usually burdens the poppet valve performance hence affects the cylinder velocity performance.

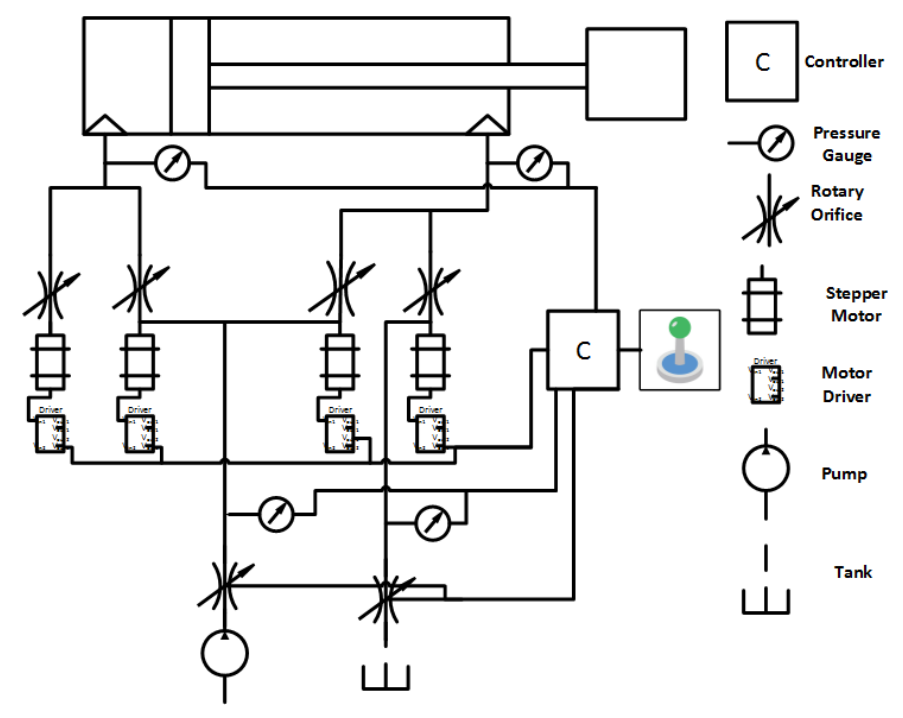

Figure 30 The schematic of Micro-Independent Metering system. [127.

\section{Independent Metering Challenges}

There are many drawbacks that prevent IM systems from spreading widely in industrial applications. In general, these challenges can be classified as follows:

1. Mode Switching. The independent metering control algorithm relies on a rule-based transition between many operating modes. This transition contributes to two main types of discontinuity which are the valves control signal and the system dynamics. The effects of the valve control signal 
disruption are a velocity oscillation and pressure peaks 28. A tracking algorithm based on online and offline controllers has been developed to reduce the effect of the control signal interruption. To overcome the dynamics problems which are the actuator motion instability and velocity oscillation, a continuous mode switching was developed by Shenouda in 28, but this solution suffers from extra power losses. However, the solutions to this problem can be listed as follows:

- Slowing the system reaction. This allows the controller to react to the pressure after instability decay, but this technique slows down the system.

- Slow down the valve using step-wise or ramp signal, but this technique slows down the system. 128.

- A dwell time technique which detects the signal and executes after enough time period that allows decaying the pressure instability. However, the efficiency of this method depends on selecting a suitable time.

2. Oscillation. The hydraulic actuator oscillation is an important point to investigate. The independent metering reduces energy losses by enlarging the orifices, but this dwindles the controllability and produces velocity oscillation due to lack of damping at the enlarged orifices. Also, the changing between different operation modes with different dynamic characteristics is another source of oscillation. A high pass filter and PD regulator are used to improve the damping and other parameters such as stability margin and vibration reduction for multi-actuator system [129.

3. Coupling. The independent metering system transfers the electrohydraulic drives into MIMO systems. For the coupling between the pressure and velocity, in the IM systems, many techniques were developed as discussed in Section 4. Each method has some shortcomings, and a new decoupling strategy is an important point to investigate. 
4. Pump/Valve Coordinate Control. The IM grants a separation control of flow and pressure which requires a coordinate control between the pump flow and the IM valves. Many iterations have been developed and one of them is to fully open the meter-out valve and control the meter-in, but this increases throttle losses by the meter-in. Another solution is to make the meter-in fully open and control the flow using the electronically controlled pump [130. This technique prevents implementing the pressure compensation method which is important for the IM systems. Another method is to fully open the meter-in and combine the control with the meter-out 131. This method relies on many modes with different dynamic characteristics, so this technique affects the system dynamics overall. This represents an important research point where further study to new systems that combine energy saving and system dynamic can be performed.

5. Safety and Reliability. Using systems including software, electronics, and smart valves increases the fault percentage of these systems 132 . This is a very important aspect to investigate. Faults in the independent metering systems can be classified into:

- Function fault: it appears if one of the IM valves lost performance or one of them encounters performance deviation. There are three main techniques developed to overcome this fault which are model-based design, intelligent control, and neural network 133. The modelbased design is not suitable for the IM applications due to the high non-linearity in fluid applications. A neural network fault detection algorithm was developed by Opendbosch in [134.

- Accuracy lost: it appears if one of the sensors lost functionality. A configurable controller is a solution used to deal with sensor fault in the IM systems, similar to what used in [135].

- Stability lost: during mode switching, the system may suffer from losing stability. Different iterations have been introduced such as 
step-wise or dwell time control. These solutions have some drawbacks that affect system dynamics. So, new methods or techniques is an important sector of IM to investigate.

Finally, as illustrated in Section 3, a new trend of IM development is to use a new stepped rotary flow control valve. This valve uses a stepper motor for rotation. A model-based study for a simple IM configuration based on this valve was included in Section 7. The new system requires different kind of analyses such as

- As inserting the stepper motor changes the valve activation concept from infinite positioning to finite one, the mathematical relation between the two valves in each IM operation mode has to be studied in a different approach of what Tabor developed in [27. Tabor, in [27, considered the mathematical relation using the poppet valve which has nonlinear and non stable performance.

- There are different driving techniques for the stepper motor which are the full step, half step and micro-step. Every type has a different effect on the valve and the IM system dynamics. This requires studying these techniques and determine the suitable valve activation technique. Moreover, developing a method that allows changing between these modes during operation without effect the system dynamics is essential.

- Develop a control algorithm that can extract new facilities for the hydraulic machine user. For example, driving the stepper motor using microstepping technique smoothness the hydraulic actuator movement. This can be one of the machine operator's options.

\section{Conclusion}

This paper introduces a comprehensive review of IM technology with its relative systems. This technology is used in a range of very important applications 

valves, such as the stepped rotary valve, and their effect on IM, from different aspects as mentioned in the paper, will lead to new and novel technologies in this field. 
[1] A. Parr, Hydraulics and pneumatics: a technician's and engineer's guide, Elsevier, 2011, Ch. 1, p. 8.

[2] J. Mattila, J. Koivumäki, D. G. Caldwell, C. Semini, A survey on control of hydraulic robotic manipulators with projection to future trends, IEEE/ASME Transactions on Mechatronics 22 (2) (2017) 669-680.

[3] K. Edge, The control of fluid power systems-responding to the challenges, Proceedings of the Institution of Mechanical Engineers, Part I: Journal of Systems and Control Engineering 211 (2) (1997) 91-110.

[4] R. Ding, J. Zhang, B. Xu, M. Cheng, Programmable hydraulic control technique in construction machinery: Status, challenges and countermeasures, Automation in Construction 95 (2018) 172-192.

[5] J. Weber, B. Beck, E. Fischer, R. Ivantysyn, G. Kolks, M. Kunkis, H. Lohse, J. Lübbert, S. Michel, M. Schneider, et al., Novel system architectures by individual drives, in: 10th International Fluid Power Conference, IFK Conference proceedings, Vol. 2, 2016.

[6] G. Yan, The design of control system for sz-40 injection molding machine base on plc, in: Mechanic Automation and Control Engineering (MACE), 2011 Second International Conference on, IEEE, 2011, pp. 7815-7818.

[7] M. Schneider, O. Koch, J. Weber, Green wheel loader-improving fuel economy through energy efficient drive and control concepts, in: Proceedings of the 10th International Fluid Power Conference (10. IFK), Dresden, Germany, 2016, pp. 8-10.

[8] R. Hippalgaonkar, M. Ivantysynova, A series-parallel hydraulic hybrid mini-excavator with displacement controlled actuators, in: 13th Scandinavian International Conference on Fluid Power; June 3-5; 2013; Linköping; Sweden, no. 092, Linköping University Electronic Press, 2013, pp. 31-42. 
[9] S. Habibi, A. Goldenberg, Design of a new high performance electrohydraulic actuator, in: Advanced Intelligent Mechatronics, 1999. Proceedings. 1999 IEEE/ASME International Conference on, IEEE, 1999, pp. $227-232$.

[10] K. K. Ahn, D. N. C. Nam, M. Jin, Adaptive backstepping control of an electrohydraulic actuator, IEEE/ASME transactions on mechatronics 19 (3) (2014) 987-995.

[11] D.-I. J. Lübbert, D.-I. A. Sitte, I. J. Weber, Pressure compensator controla novel independent metering architecture, in: 10th International Fluid Power Conference, Vol. 1, 2016, pp. 231-245.

[12] B. Eriksson, J.-O. Palmberg, Individual metering fluid power systems: challenges and opportunities, Proceedings of the institution of mechanical engineers, part I: journal of systems and control engineering 225 (2) (2011) $196-211$.

[13] S. Liu, B. Yao, Energy-saving control of single-rod hydraulic cylinders with programmable valves and improved working mode selection, Tech. rep., SAE Technical Paper (2002).

[14] X. Kong, D. Shan, J. Yao, Y. Gao, Study on experiment and modeling for the multifunctional integrated valve control system, in: Intelligent Mechatronics and Automation, 2004. Proceedings. 2004 International Conference on, IEEE, 2004, pp. 455-459.

[15] B. Eriksson, Control strategy for energy efficient fluid power actuators: Utilizing individual metering, Ph.D. thesis, Linköping University Electronic Press (2007).

[16] A. Sitte, J. Weber, Structural design of independent metering control systems, in: 13th Scandinavian International Conference on Fluid Power; June 3-5; 2013; Linköping; Sweden, no. 092, Linköping University Electronic Press, 2013, pp. 261-270. 
[22] B. K. Nielsen, Controller development for a separate meter-in separate meter-out fluid power valve for mobile applications, Ph.D. thesis, Citeseer (2005).

[23] J. Mattila, T. Virvalo, Energy-efficient motion control of a hydraulic ma-

[17] A. Jansson, P. Krus, J.-O. Palmberg, Decoupling of response and pressure level in a hydraulic actuator, in: The 4th Bath International Fluid Power Workshop, 1991.

[18] P. Opdenbosch, N. Sadegh, W. Book, A. Enes, Auto-calibration based control for independent metering of hydraulic actuators, in: Robotics and Automation (ICRA), 2011 IEEE International Conference on, IEEE, 2011, pp. 153-158.

[19] K. Choi, J. Seo, Y. Nam, K. U. Kim, Energy-saving in excavators with application of independent metering valve, Journal of Mechanical Science and Technology 29 (1) (2015) 387-395.

[20] M. Vukovic, H. Murrenhoff, Single edge meter out control for mobile machinery, in: ASME/BATH 2014 Symposium on Fluid Power and Motion Control, American Society of Mechanical Engineers, 2014, pp. V001T01A009-V001T01A009.

[21] A. Jansson, J.-O. Palmberg, Separate controls of meter-in and meter-out orifices in mobile hyraulic systems, SAE transactions (1990) 377-383. nipulator, in: Robotics and Automation, 2000. Proceedings. ICRA'00. IEEE International Conference on, Vol. 3, IEEE, 2000, pp. 3000-3006.

[24] H. Hu, Q. Zhang, Multi-function realization using an integrated programmable e/h control valve, Applied engineering in agriculture 19 (3) (2003) 283.

[25] B. Yao, C. DeBoer, Energy-saving adaptive robust motion control of single-rod hydraulic cylinders with programmable valves, in: American 
Control Conference, 2002. Proceedings of the 2002, Vol. 6, IEEE, 2002, pp. $4819-4824$.

[26] L. Lu, B. Yao, Energy-saving adaptive robust control of a hydraulic manipulator using five cartridge valves with an accumulator, IEEE Transactions on Industrial Electronics 61 (12) (2014) 7046-7054.

[27] K. A. Tabor, Optimal velocity control and cavitation prevention of a hydraulic actuator using four valve independent metering, Tech. rep., SAE Technical Paper (2005).

[28] A. Shenouda, Quasi-static hydraulic control systems and energy savings potential using independent metering four-valve assembly configuration, Ph.D. thesis, Georgia Institute of Technology (2006).

[29] M. S. A. Laamanen, M. Vilenius, Is it time for digital hydraulics, in: The Eighth Scandinavian International Conference on Fluid Power, 2003.

[30] M. Ketonen, M. Linjama, High flowrate digital hydraulic valve system, in: Proc. of The Ninth Workshop on Digital Fluid Power, Aalborg, Denmark, 2017.

[31] M. Vukovic, H. Murrenhoff, The next generation of fluid power systems, Procedia engineering 106 (2015) 2-7.

[32] M. Vukovic, R. Leifeld, H. Murrenhoff, Steam-a hydraulic hybrid architecture for excavators, in: 10th International Fluid Power Conference (10. IFK), 2016, pp. 8-10.

[33] M. Linjama, M. Paloniitty, L. Tiainen, K. Huhtala, Mechatronic design of digital hydraulic micro valve package, Procedia engineering 106 (2015) $97-107$.

[34] M. Linjama, M. Huova, O. Karhu, K. Huhtala, High-performance digital hydraulic tracking control of a mobile boom mockup, in: 10th International Fluid Power Conference (10. IFK), 2016, p. 12. 
[35] M. Heikkila, Energy Efficient Boom Actuation Using a Digital Hydraulic

[ [36] J. control of hydraulic robots, Control Engineering Practice 85 (2019) 176 193. doi:https://doi.org/10.1016/j.conengprac.2018.12.013. URL S0967066118307238

[37] M. Axin, Fluid power systems for mobile applications: With a focus on energy efficiency and dynamic characteristics, Ph.D. thesis, Linköping University Electronic Press (2013).

740

[38] A. DellAmico, M. Carlsson, E. Norlin, M. Sethson, Investigation of a digital hydraulic actuation system on an excavator arm, in: 13th Scandinavian International Conference on Fluid Power; June 3-5; 2013; Linköping; Sweden, no. 092, Linköping University Electronic Press, 2013, pp. 505-511.

[39] J. de Brun Mangs, M. Tillquist, Evaluation of a programmable hydraulic

[40] J. Lodewyks, P. Zurbrügg, Decentralized energy-saving hydraulic concepts for mobile working machines, in: Proc. IFK, 2016, pp. 79-90.

q [41] P. Opdenbosch, N. Sadegh, W. Book, T. Murray, R. Yang, Modelling an electro-hydraulic poppet valve, International Journal of Fluid Power 10 (1) (2009) 7-15. arXiv:https://doi.org/10.1080/14399776.2009. 10780963, doi:10.1080/14399776.2009.10780963.

URL https://doi.org/10.1080/14399776.2009.10780963

[42] P. Opdenbosch, N. Sadegh, W. Book, Learning control applied to electrohydraulic poppet valves, in: 2008 American Control Conference, 2008, pp. 
[ [43] P. Opdenbosch, N. Sadegh, W. Book, Intelligent controls for electrohydraulic poppet valves, Control Engineering Practice 21 (6) (2013) 789 - 796. doi:https://doi.org/10.1016/j.conengprac.2013.02.008.

(1) URL http://www.sciencedirect.com/science/article/pii/ S0967066113000269

[44] A. Shenouda, W. Book, Energy saving analysis using a four-valve independent metering configuration controlling a hydraulic cylinder, in: SAE Technical Paper, SAE International, 2005. doi:10.4271/2005-01-3632. URL https://doi.org/10.4271/2005-01-3632

${ }_{765}$ [ [45] A. Shenouda, W. Book, Optimal mode switching for a hydraulic actuator controlled with four-valve independent metering configuration, International Journal of Fluid Power 9 (1) (2008) 35-43. arXiv:https://

(1) doi.org/10.1080/14399776.2008.10781295 doi:10.1080/14399776. 2008.10781295 . URL https : //doi .org/10.1080/14399776.2008.10781295

[46] R. Ding, B. Xu, J. Zhang, M. Cheng, Bumpless mode switch of independent metering fluid power system for mobile machinery, Automation in Construction 68 (2016) 52-64.

[47] B. Xu, R. Ding, J. Zhang, M. Cheng, T. Sun, Pump/valves coordinate control of the independent metering system for mobile machinery, $\mathrm{Au}-$ tomation in Construction 57 (2015) 98-111.

口 [48] R. Ding, J. Zhang, B. Xu, M. Cheng, M. Pan, Energy efficiency improvea ment of heavy-load mobile hydraulic manipulator with electronically tunable operating modes, Energy Conversion and Management 188 (2019) 447 - 461. doi:https://doi.org/10.1016/j.enconman.2019.03.023. URL http://www.sciencedirect.com/science/article/pii/ S0196890419303073

[49] F. Campanini, R. Bianchi, A. Vacca, P. Casoli, Optimized control for an independent metering valve with integrated diagnostic features, in: 
q [55] R. Zhang, A. G. Alleyne, E. A. Prasetiawan, Performance limitations of a class of two-stage electro-hydraulic flow valves, International Journal

[

[56] H. Murrenhoff, S. Sgro, M. Vukovic, An overview of energy saving architectures for mobile applications, in: Proceedings of the 9th International Fluid Power Conference, Aachen, Germany, 2014, pp. 24-26.

[57] P. Dengler, J. Groh, M. Geimer, Valve control concepts in a constant Conference on Hydraulics and Pneumatics, 2011. 
[58] P. Krus, On load sensing fluid power systems, Division of Fluid Power Control Department of Mechanical Engineering, Linkoping University, Sweden.

[59] Y. Sakurai, T. Nakada, K. Tanaka, Design method of an intelligent oilhydraulic system (load sensing oil-hydraulic system), in: Intelligent Control, 2002. Proceedings of the 2002 IEEE International Symposium on, IEEE, 2002, pp. 626-630.

[60] R. H. Hansen, Advanced power management of a telehandler using electronic load sensing, in: 10th international workshop on research and education in mechatronics, University of Strathclyde Glasgow, UK, 2009.

[61] D. Lovrec, M. Kastrevc, S. Ulaga, Electro-hydraulic load sensing with a speed-controlled hydraulic supply system on forming-machines, The International Journal of Advanced Manufacturing Technology 41 (11-12) (2009) 1066-1075.

[62] A. Lettini, M. Havermann, M. Guidetti, A. Fornaciari, Electro-hydraulic load sensing: A contribution to increased efficiency through fluid power on mobile machines, in: Proceedings of the 7. IFK: International Fluid Power Conference, Aachen, Germany, 2010, pp. 22-24.

[63] R. S. Jackson, R. R. Clanton, J. L. Pfaff, Hydraulic control valve system with electronic load sense control, uS Patent 7,089,733 (Aug. 15 2006).

[64] T. W. Dell (Ed.), Hydraulic systems for mobile equipment, Vol. 1, Ch. Load sensing pressure compensating (LSPC) hydraulic system, pp. 417455 .

[65] M. Scherer, M. Geimer, B. Weiss, Contribution on control strategies of flow-on-demand hydraulic circuits, in: 13th Scandinavian International Conference on Fluid Power; June 3-5; 2013; Linköping; Sweden, no. 092, Linköping University Electronic Press, 2013, pp. 531-540. 
[66] D. P. Smith, D. T. Mather, Hydraulic regeneration system, uS Patent

[74] M. Karvonen, M. Heikkilä, M. Huova, M. Linjama, Analysis by simulation of different control algorithms of a digital hydraulic two-actuator system, International journal of fluid power 15 (1) (2014) 33-44.

[75] M. Vukovic, S. Sgro, H. Murrenhoff, Steam: A mobile hydraulic system 865

[67] K. Liu, Y. Gao, Z. Tu, Energy saving potential of load sensing system with hydro-mechanical pressure compensation and independent metering, International Journal of Fluid Power 17 (3) (2016) 173-186.

[68] A. J. Kleitsch, Independent metering valve with flow sharing, uS Patent 2017/0108015 (Apr. 20 2017).

[69] X. Huang, S. V. Lunzman, Electro-hydraulic valve control system and method, uS Patent 6,662,705 (Dec. 16 2003).

[70] J. A. Aardema, D. W. Koehler, System and method for controlling an independent metering valve, uS Patent 5,947,140 (Sep. 7 1999).

[71] M. Huova, Energy efficient digital hydraulic valve control, Tampereen teknillinen yliopisto. Julkaisu-Tampere University of Technology. Publication; 1298.

[72] M. Heikkilä, M. Linjama, Displacement control of a mobile crane using a digital hydraulic power management system, Mechatronics 23 (4) (2013)

[73] M. Ketonen, M. Linjama, Simulation study of a digital hydraulic independent metering valve system on an excavator, in: Proceedings of 15: th Scandinavian International Conference on Fluid Power, June 7-9, 2017, Linköping, Sweden, no. 144, Linköping University Electronic Press, 2017, pp. 136-146.

with engine integration, in: ASME/BATH 2013 Symposium on Fluid 
Power and Motion Control, American Society of Mechanical Engineers, 2013, pp. V001T01A005-V001T01A005.

[76] M. Vukovic, S. Sgro, H. Murrenhoff, Steam-a holistic approach to designing excavator systems, in: Proceedings of the 9th International Fluid Power Conference, Aachen, Germany, 2014, pp. 24-26.

[77] B. Eriksson, Mobile fluid power systems design: with a focus on energy efficiency, Ph.D. thesis, Linköping University Electronic Press (2010).

[78] J. A. Crosser, Hydraulic circuit and control system therefor, uS Patent 5,138,838 (Aug. 18 1992).

[79] D. P. Smith, D. T. Mather, Electro-hydraulic metering valve with integral flow control, uS Patent 7,240,604 (Jul. 10 2007).

[80] D. P. Smith, Electrohydraulic valve arrangement, uS Patent 5,868,059 (Feb. 9 1999).

[81] T. J. Hajek Jr, S. T. Tolappa, Independent and regenerative mode fluid control system, uS Patent 6,715,403 (Apr. 6 2004).

[82] S. Liu, B. Yao, Programmable valves: a solution to bypass deadband problem of electro-hydraulic systems, in: American Control Conference, 2004. Proceedings of the 2004, Vol. 5, IEEE, 2004, pp. 4438-4443.

[83] R. Zhang, A. G. Alleyne, E. A. Prasetiawan, Performance limitations of a class of two-stage electro-hydraulic flow valves, International Journal of Fluid Power 3 (1) (2002) 47-53.

[84] S. Liu, G. Krutz, B. Yao, Easy5 model of two position solenoid operated cartridge valve, in: ASME 2002 International Mechanical Engineering Congress and Exposition, American Society of Mechanical Engineers, 2002, pp. 63-67.

[85] B. Yoo, E. C. Hughes, R. D. Vance, Method for calibrating independent metering valves, uS Patent 7,562,554 (Jul. 21 2009). 
[86] EATON, Ultorincs ZTS16 Twin Spool Valve, Technical Catalog 1, EATON Powering Business Worldwide (January 2010).

[87] M. Rannow, Fail operational controls for an independent metering valve, in: Proceedings of the 10. International Fluid Power Conference, 2016.

[88] M. Karvonen, Energy efficient digital hydraulic power management of a multi actuator system, English. PhD thesis. Tampere University of Technology (2016) 16-29.

[89] EATON, EPV10 - Proportional Valve (12 2019).

[90] BUCHER, 2/2 Cartridge Seat Valve, Size 5 (9 2015).

[91] EATON, CMA90 Advanced Independent-Metering Mobile Valve (9 2016).

[92] EATON, CMA200 Advanced Independent-Metering Mobile Valve (10 2016).

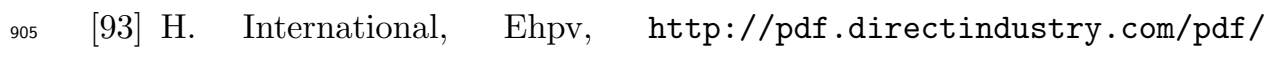
husco-international/ehpv/117113-608498.html (2019).

[94] I. Okhotnikov, S. Noroozi, P. Sewell, P. Godfrey, Evaluation of steady flow torques and pressure losses in a rotary flow control valve by means of computational fluid dynamics, International Journal of Heat and Fluid Flow 64 (2017) 89-102.

[95] K. Abuowda, S. Noroozi, M. Dupac, P. Godfry, A dynamic model and performance analysis of a stepped rotary flow control valve, Proceedings of the Institution of Mechanical Engineers, Part I: Journal of Systems and Control Engineering (2019) 0959651818820978.

915

[96] K. Abuowda, S. Noroozi, M. Dupac, P. Godfrey, Friction analysis and modelling of a novel stepped rotary flow control valve, in: The Fifteenth International Conference on Condition Monitoring and Machinery Failure Prevention Technologies CM/MFPT 2018, p. 9781538653456. 
[97] K. Abuowda, S. Noroozi, M. Dupac, P. Godfrey, Sensor-less control of a novel stepped hydraulic flow control valve, in: The Fifteenth International Conference on Condition Monitoring and Machinery Failure Prevention Technologies CM/MFPT 2018, p. 9781538653456.

[98] H. C. Pedersen, T. O. Andersen, T. Skouboe, M. S. Jacobsen, Investigation and comparison of separate meter-in separate meter-out control strategies, in: ASME/BATH 2013 Symposium on Fluid Power and Motion Control, American Society of Mechanical Engineers, 2013, pp. V001T01A047V001T01A047.

[99] G. Kolks, J. Weber, Modiciency-efficient industrial hydraulic drives through independent metering using optimal operating modes, in: Proceedings of the 10th International Conference on Fluid Power, 2016.

[100] G. Kolks, J. Weber, Controller design for precise and efficient industrial cylinder drives using independent metering valves, in: 9th FPNI Ph. D. Symposium on Fluid Power, American Society of Mechanical Engineers, 2016, pp. V001T01A009-V001T01A009.

[101] G. Rath, E. Zaev, Optimal control for hydraulic system with separate meter-in and separate meter-out, in: Proceedings of the 15th Scandinavian International Conference on Fluid Power, SICFP17, 2017.

[102] Q. Zhong, B. Zhang, M. Niu, H. Hong, H. Yang, Research on dynamic performance of independent metering control system, in: ASME/BATH 2017 Symposium on Fluid Power and Motion Control, American Society of Mechanical Engineers, 2017, pp. V001T01A006-V001T01A006.

[103] A. H. Hansen, H. C. Pedersen, T. O. Andersen, L. Wachmann, Design of energy efficient smismo-els control strategies, in: Fluid Power and Mechatronics (FPM), 2011 International Conference on, IEEE, 2011, pp. 522527. 
[104] M. Borghi, B. Zardin, F. Pintore, F. Belluzzi, Energy savings in the hydraulic circuit of agricultural tractors, Energy Procedia 45 (2014) 352-361.

[105] J. Zhang, S. Jiao, X. Liao, P. Yin, Y. Wang, K. Si, Y. Zhang, H. Gu, Design of intelligent hydraulic excavator control system based on pid method, in: International Conference on Computer and Computing Technologies in Agriculture, Springer, 2009, pp. 207-215.

[106] J. Watton, Fundamentals of fluid power control, Vol. 10, Cambridge University Press, 2009, p. 494.

[107] S. Liu, B. Yao, Coordinate control of energy saving programmable valves, IEEE Transactions on Control Systems Technology 16 (1) (2008) 34-45.

[108] G. Chen, J. Wang, S. Wang, J. Zhao, W. Shen, Energy saving control in separate meter in and separate meter out control system, Control Engineering Practice 72 (2018) 138-150.

[109] G. Chen, J. Wang, S. Wang, J. Zhao, W. Shen, Indirect adaptive robust dynamic surface control in separate meter-in and separate meter-out control system, Nonlinear Dynamics 90 (2) (2017) 951-970.

[110] R. Ding, B. Xu, J. Zhang, M. Cheng, Self-tuning pressure-feedback control by pole placement for vibration reduction of excavator with independent metering fluid power system, Mechanical Systems and Signal Processing 92 (2017) 86-106.

[111] A. Shenouda, W. J. Book, Selection of operating modes of a multifunctional hydraulic device, in: ASME 2005 International Mechanical Engineering Congress and Exposition, American Society of Mechanical Engineers, 2005, pp. 99-109.

[112] S. N. Alkam, New methods in modeling and control of modern electrohydraulic systems, Ph.D. thesis (2014). 
[113] H. YUAN, Y. SHANG, M. VUKOVIC, S. WU, H. MURRENHOFF, Z. JIAO, Characteristics of energy efficient switched hydraulic systems, JFPS International Journal of Fluid Power System 8 (2) (2014) 90-98.

[120] J.-C. Lee, K.-C. Jin, Y.-M. Kwon, L.-G. Choi, J.-Y. Choi, B.-K. Lee, Development of the independent metering valve control system and analysis of its performance for an excavator, in: BATH/ASME 2016 Symposium 
on Fluid Power and Motion Control, American Society of Mechanical En-

[121] M. Axin, Mobile working hydraulic system dynamics, Vol. 1697, Linköping University Electronic Press, 2015.

[122] M. Wydra, M. Geimer, B. Weiss, An approach to combine an independent metering system with an electro-hydraulic flow-on-demand hybrid-system, gineers, 2016, pp. V001T01A021-V001T01A021. in: Proceedings of 15: th Scandinavian International Conference on Fluid Power, June 7-9, 2017, Linköping, Sweden, no. 144, Linköping University Electronic Press, 2017, pp. 161-170.

[123] L. Ge, L. Quan, X. Zhang, B. Zhao, J. Yang, Efficiency improvement and evaluation of electric hydraulic excavator with speed and displacement variable pump, Energy conversion and management 150 (2017) 62-71.

[124] J. D. Linerode, Swing control algorithm for hydraulic circuit, uS Patent 6,761,029 (Jul. 13 2004).

[125] B. Thompson, H.-S. Yoon, J. Kim, J. Lee, Swing energy recuperation scheme for hydraulic excavators, Tech. rep., SAE Technical Paper (2014).

[126] W. Huang, L. Quan, J. Huang, J. Yang, Flow matching with combined control of the pump and the valves for the independent metering swing system of a hydraulic excavator, Proceedings of the Institution of Mechanical Engineers, Part D: Journal of Automobile Engineering 232 (10) (2018) 1310-1322.

[127] K. Abuowda, S. Noroozi, M. Dupac, P. Godfrey, Algorithm design for the novel mechatronics electro-hydraulic driving system: Micro-independent metering, in: 2019 IEEE International Conference on Mechatronics (ICM), Vol. 1, IEEE, 2019, pp. 7-12.

[128] M. Linjama, Digital fluid power: State of the art, in: 12th Scandinavian International Conference on Fluid Power, Tampere, Finland, May, 2011, pp. $18-20$. 
[129] M. Cheng, J. Zhang, B. Xu, R. Ding, J. Wei, Decoupling compensation for damping improvement of the electrohydraulic control system with multiple actuators, IEEE/ASME Transactions on Mechatronics 23 (3) (2018) 13831392.

[130] B. Liu, L. Quan, L. Ge, Research on the performance of hydraulic excavator boom based pressure and flow accordance control with independent metering circuit, Proceedings of the Institution of Mechanical Engineers, Part E: Journal of Process Mechanical Engineering 231 (5) (2017) 901913.

[131] R. Ding, J. Zhang, B. Xu, Advanced energy management of a novel independent metering meter-out control system: A case study of an excavator, IEEE Access 6 (2018) 45782-45795.

[132] J. Weber, Independent metering systems, International Journal of Hydromechatronics 1 (1) (2018) 91-106.

[133] M. Bian, J. Shi, S. Wang, Fta-based fault diagnose expert system for hydraulic equipments, in: Proceedings of 2011 International Conference on Fluid Power and Mechatronics, IEEE, 2011, pp. 959-963.

[134] P. Opdenbosch, N. Sadegh, W. Book, Intelligent controls for electrohydraulic poppet valves, Control Engineering Practice 21 (6) (2013) 789796.

[135] L. Siivonen, M. Linjama, M. Huova, M. Vilenius, Jammed on/off valve fault compensation with distributed digital valve system, International Journal of Fluid Power 10 (2) (2009) 73-82. 